\title{
GLOBAL RIGIDITY OF 2-DIMENSIONAL LINEARLY CONSTRAINED FRAMEWORKS
}

\author{
HAKAN GULER, BILL JACKSON, AND ANTHONY NIXON
}

\begin{abstract}
A linearly constrained framework in $\mathbb{R}^{d}$ is a point configuration together with a system of constraints which fixes the distances between some pairs of points and additionally restricts some of the points to lie in given affine subspaces. It is globally rigid if the configuration is uniquely defined by the constraint system, and is rigid if it is uniquely defined within some small open neighbourhood. Streinu and Theran characterised generic rigidity of linearly constrained frameworks in $\mathbb{R}^{2}$ in 2010 . We obtain an analagous characterisation for generic global rigidity in $\mathbb{R}^{2}$. More precisely we show that a generic linearly constrained framework in $\mathbb{R}^{2}$ is globally rigid if and only if it is redundantly rigid and 'balanced'. For generic frameworks which are not balanced, we determine the precise number of solutions to the constraint system whenever the underlying rigidity matroid of the given framework is connected. We also obtain a stress matrix sufficient condition and a Hendrickson type necessary condition for a generic linearly constrained framework to be globally rigid in $\mathbb{R}^{d}$.
\end{abstract}

\section{INTRODUCTION}

A (bar-joint) framework $(G, p)$ in $\mathbb{R}^{d}$ is the combination of a finite, simple graph $G=$ $(V, E)$ and a realisation $p: V \rightarrow \mathbb{R}^{d}$. The framework $(G, p)$ is rigid if every edge-length preserving continuous motion of the vertices arises as a congruence of $\mathbb{R}^{d}$. Moreover $(G, p)$ is globally rigid if every framework $(G, q)$ with the same edge lengths as $(G, p)$ arises from a congruence of $\mathbb{R}^{d}$.

In general it is an NP-hard problem to determine the global rigidity of a given framework [21. The problem becomes more tractable, however, if we consider generic frameworks i.e. frameworks in which the set of coordinates of the points is algebraically independent over $\mathbb{Q}$. Hendrickson 10 obtained two necessary conditions for a generic framework $(G, p)$ in $\mathbb{R}^{d}$ to be globally rigid: the graph $G$ should be $(d+1)$-connected, and the framework $(G, p)$ should be redundantly rigid i.e. it remains rigid after deleting any edge. While Hendrickson's conditions are insufficient to imply generic global rigidity when $d \geq 3$ [4, 18], they are sufficient when $d=1,2$. In particular, we have the following theorem of Jackson and Jordán [1] when $d=2$.

Theorem 1.1. A generic framework $(G, p)$ in $\mathbb{R}^{2}$ is globally rigid if and only if $G$ is either a complete graph on at most three vertices or $G$ is 3-connected and redundantly rigid.

A linearly constrained framework is a bar-joint framework in which certain vertices are constrained to lie in given affine subspaces, in addition to the usual distance constraints between pairs of vertices. Linearly constrained frameworks are motivated by numerous practical applications, notably in mechanical engineering and biophysics, see for example

Date: July 18, 2019.

2010 Mathematics Subject Classification. 52C25, 05C10 and 53A05.

Key words and phrases. rigidity, global rigidity, stress matrix, sliders, linearly constrained framework, count matroid. 
[9, 23]. Streinu and Theran [22] give a characterisation for generic rigidity of linearly constrained frameworks in $\mathbb{R}^{2}$. Together with Cruickshank [8], we recently obtained an analogous characterisation for generic rigidity of linearly constrained frameworks in $\mathbb{R}^{d}$ as long as the dimensions of the affine subspaces at each vertex are sufficiently small (compared to $d$ ). In this article we consider global rigidity for linearly constrained frameworks. Global rigidity of bar-joint frameworks has its own suite of practical applications, for example in sensor network localisation [13], and we expect our extension to have similar uses.

Throughout this paper we will consider graphs whose only possible multiple edges are multiple loops. We call such a graph $G=(V, E, L)$ a looped simple graph where $E$ denotes the set of (non-loop) edges and $L$ the set of loops. A $d$-dimensional linearly constrained framework is a triple $(G, p, q)$ where $G=(V, E, L)$ is a looped simple graph, $p: V \rightarrow \mathbb{R}^{d}$ and $q: L \rightarrow \mathbb{R}^{d}$. For $v_{i} \in V$ and $e_{j} \in L$ we put $p\left(v_{i}\right)=p_{i}$ and $q\left(e_{j}\right)=q_{j}$. The framework $(G, p, q)$ is generic if the set of coordinates of $\{p, q\}$ is algebraically independent over $\mathbb{Q}$ i.e. the transcendence degree of $\mathbb{Q}(p, q)$ over $\mathbb{Q}$ is $d(|V|+|L|)$.

Two $d$-dimensional linearly constrained frameworks $(G, p, q)$ and $(G, \tilde{p}, q)$ are equivalent if

$$
\begin{aligned}
\left\|p_{i}-p_{j}\right\|^{2} & =\left\|\tilde{p}_{i}-\tilde{p}_{j}\right\|^{2} \text { for all } v_{i} v_{j} \in E, \text { and } \\
p_{i} \cdot q_{j} & =\tilde{p}_{i} \cdot q_{j} \text { for all incident pairs } v_{i} \in V \text { and } e_{j} \in L .
\end{aligned}
$$

We say that $(G, p, q)$ is globally rigid if its only equivalent framework is itself.

We give an illustration of rigidity and global rigidity in $\mathbb{R}^{2}$ in Figure 1, First note that a loop at a vertex constrains that vertex to lie on a specific line. Every realisation of the graph $H$ as a generic linearly constrained framework will be globally rigid as having two different line constraints at each vertex fixes the position of the vertices in $\mathbb{R}^{2}$. Every generic realisation $(G, p)$ of the graph $G$ is rigid by Theorem 2.1 below, but is not globally rigid, since we can obtain an equivalent realisation by reflecting the vertex $v_{2}$ in the line through $p\left(v_{1}\right)$ which is perpendicular to the line constraint at $v_{2}$.
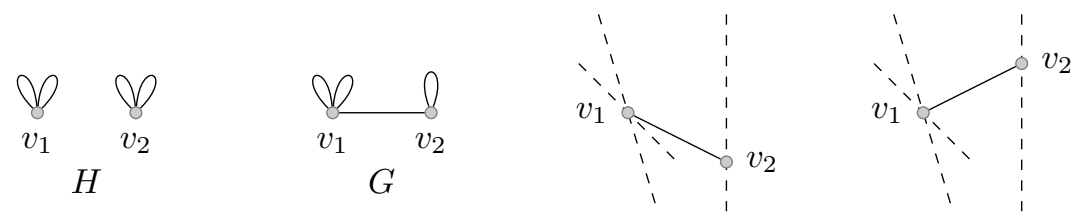

Figure 1. Every realisation of the graph $H$ as a generic linearly constrained framework in $\mathbb{R}^{2}$ is globally rigid. Every generic realisation of the graph $G$ is rigid but not globally rigid. Two distinct equivalent realisations of $G$ are given on the right of the figure.

Our main results characterise global rigidity for generic linearly constrained frameworks in $\mathbb{R}^{2}$ and determine the precise number of frameworks which are equivalent to a given generic framework whenever the underlying rigidity matroid of the given framework is connected. We also obtain a stress matrix sufficient condition and a Hendrickson type necessary condition for generic global rigidity in $\mathbb{R}^{d}$. A more detailed description of the content of the paper is as follows.

In Section 2 we provide a brief background on infinitesimal rigidity for linearly constrained frameworks. In Section 3 we give necessary conditions for generic global rigidity analogous to Hendrickson's conditions. We obtain an algebraic sufficient condition for the generic global rigidity of a linearly constrained framework in Section 4. This sufficient condition in 
terms of the rank of an appropriate stress matrix extends a key result of Connelly [5] for barjoint frameworks. We focus on characterising generic global rigidity in $\mathbb{R}^{2}$ in the remainder of the paper. We obtain structural results on rigid circuits in the generic 2-dimensional linearly constrained rigidity matroid in Section [5. These are used in Sections 6 and 7 to obtain a recursive construction for the family of looped simple graphs which are balanced and generically redundantly rigid. The recursive construction is then used in Section 8 to characterise generic global rigidity. We determine the precise number of frameworks which are equivalent to a given generic framework whenever the underlying rigidity matroid of the given framework is connected in Section 9 ,

\section{INFINITESIMAL RIGIDITY}

An infinitesimal motion of a linearly constrained framework $(G, p, q)$ is a map $\dot{p}: V \rightarrow \mathbb{R}^{d}$ satisfying the system of linear equations:

$$
\begin{aligned}
\left(p_{i}-p_{j}\right) \cdot\left(\dot{p}_{i}-\dot{p}_{j}\right) & =0 \text { for all } v_{i} v_{j} \in E \\
q_{j} \cdot \dot{p}_{i} & =0 \text { for all incident pairs } v_{i} \in V \text { and } e_{j} \in L .
\end{aligned}
$$

The second constraint implies that, for each vertex $v_{i}$, its infinitesimal velocity $\dot{p}\left(v_{i}\right)$ is constrained to lie in the intersection of the hyperplanes with normals $q_{j}$ for every loop $e_{j}$ incident to $v_{i}$.

The rigidity matrix $R(G, p, q)$ of the framework is the matrix of coefficients of this system of equations for the unknowns $\dot{p}$. Thus $R(G, p, q)$ is a $(|E|+|L|) \times d|V|$ matrix, in which: the row indexed by an edge $v_{i} v_{j} \in E$ has $p(u)-p(v)$ and $p(v)-p(u)$ in the $d$ columns indexed by $v_{i}$ and $v_{j}$, respectively and zeros elsewhere; the row indexed by a loop $e_{j}=v_{i} v_{i} \in L$ has $q_{j}$ in the $d$ columns indexed by $v_{i}$ and zeros elsewhere.

The framework $(G, p, q)$ is infinitesimally rigid if its only infinitesimal motion is $\dot{p}=$ 0 , or equivalently if $\operatorname{rank} R(G, p, q)=d|V|$. We say that the graph $G$ is rigid in $\mathbb{R}^{d}$ if $\operatorname{rank} R(G, p, q)=d|V|$ for some realisation $(G, p, q)$ in $\mathbb{R}^{d}$, or equivalently if $\operatorname{rank} R(G, p, q)=$ $d|V|$ for all generic realisations $(G, p, q)$.

Streinu and Theran 22 characterised the looped simple graphs $G$ which are rigid in $\mathbb{R}^{2}$. Given a looped simple graph $G=(V, E, L)$ and $F \subseteq E \cup L$, let $V_{F}$ denote the set of vertices incident to $F$.

Theorem 2.1. Let $H$ be a looped simple graph. Then $H$ is rigid in $\mathbb{R}^{2}$ if and only if $H$ has a spanning subgraph $G=(V, E, L)$ such that $|E|+|L|=2|V|,|F| \leq 2\left|V_{F}\right|$ for all $F \subseteq E \cup L$ and $|F| \leq 2\left|V_{F}\right|-3$ for all $\emptyset \neq F \subseteq E$.

\section{NECESSARY CONDITIONS FOR GLOBAL RIGIDITY}

We say that a looped graph $G=(V, E, L)$ is redundantly rigid if $G-e$ is rigid for any $e \in E \cup L$, and that $G$ is d-balanced if, for all $X \subset V$ with $|X|=d$, each connected component of $G-X$ has at least one loop. We will show that the properties of being redundantly rigid and $d$-balanced are necessary conditions for a connected generic linearly constrained framework with at least two vertices to be globally rigid in $\mathbb{R}^{d}$. (Note that a framework with one vertex is globally rigid if and only if it is rigid, and that a disconnected framework is globally rigid if and only if each of its connected components is globally rigid.)

Given a linearly constrained framework $(G, p, q)$ in $\mathbb{R}^{d}$ we define its configuration space $C(G, p, q)$ to be the set

$$
C(G, p, q)=\left\{\hat{p} \in \mathbb{R}^{d|V|}:(G, \hat{p}, q) \text { is equivalent to }(G, p, q)\right\} .
$$


In order to establish that globally rigid linearly constrained frameworks are also redundantly rigid an important step is to prove that the configuration space is compact. Since it is easy to see that the configuration space is closed this will follow from the following lemma.

Lemma 3.1. Let $(G, p, q)$ be a generic linearly constrained framework in $\mathbb{R}^{d}$. Then $C(G, p, q)$ is bounded if and only if each connected component of $G$ contains at least $d$ loops.

Proof. Let $H$ be a connected component of $G$.

Suppose $H$ does not contain $d$ loops. Let $W$ be subspace of $\mathbb{R}^{d}$ spanned by the vectors $q(f)$ for $f \in L(H)$. Choose $0 \neq t \in W^{\perp}$ and define $\left(G, p^{\prime}, q\right)$ by putting $p^{\prime}(v)=p(v)+t$ for all $v \in V(H)$, and $p^{\prime}(v)=p(v)$ for all $v \in V(G) \backslash V(H)$. Then $\left(G, p^{\prime}, q\right)$ is equivalent to $(G, p, q)$ and since we can choose $t$ to be arbitrarily large, $C(G, p, q)$ is not bounded.

Suppose $H$ contains $d$ loops $f_{1}, f_{2}, \ldots, f_{d}$. Let $B:=\sum_{x y \in E(H)}|p(x)-p(y)|$ and choose $v \in V(H)$. Then the fact that $H$ is connected implies that $\left|p(v) \cdot q\left(f_{i}\right)\right| \leq B$ for all $1 \leq i \leq d$. Since $q$ is generic we have $\mathbf{e}_{\mathbf{1}}=(1,0, \ldots, 0)=\sum_{i=1}^{d} \alpha_{i} q\left(f_{i}\right)$ for some scalars $\alpha_{1}, \alpha_{2}, \ldots, \alpha_{d}$. Hence

$$
\left|p(v) \cdot \mathbf{e}_{\mathbf{1}}\right|=\left|\sum_{i=1}^{d} \alpha_{i} p(v) \cdot q\left(f_{i}\right)\right| \leq B \sum_{i=1}^{d}\left|\alpha_{i}\right| .
$$

A similar argument shows that $\left|p(v) \cdot \mathbf{e}_{\mathbf{j}}\right|$ is bounded for all vectors $\mathbf{e}_{\mathbf{j}}$ in the standard basis for $\mathbb{R}^{d}$. Since $v$ is arbitrary, $C\left(H,\left.p\right|_{H},\left.q\right|_{H}\right)$ is bounded.

We may apply the same argument to each connected component of $G$ to deduce that $C(G, p, q)$ is bounded.

Theorem 3.2. Suppose $(G, p, q)$ is a generic globally rigid linearly constrained framework in $\mathbb{R}^{d}$. Then each connected component of $G$ is either a single vertex with at least $d$ loops or is d-balanced and redundantly rigid in $\mathbb{R}^{d}$.

Proof. Since $(G, p, q)$ is globally rigid if and only if each of its connected components are globally rigid, we may assume that $G$ is connected and is rigid in $\mathbb{R}^{d}$. It is easy to see that the theorem holds when $G$ has one vertex so we may assume that $|V| \geq 2$.

We first prove that $G$ is $d$-balanced. Let $X \subseteq V$ with $|X|=d$. Suppose some connected component $G_{1}$ of $G-X$, is incident with no loops. Then we can obtain an equivalent but noncongruent realisation from $(G, p, q)$ by reflecting $G_{1}$ in the hyperplane spanned by the points $p(v), v \in X$. This contradicts the global rigidity of $(G, p, q)$. Hence $G$ is $d$-balanced.

We next show that $G$ is redundantly rigid in $\mathbb{R}^{d}$. Suppose not. Then there exists $e \in E \cup L$ such that $(G-e, p, q)$ is flexible.

We will use Lemma 3.1 to show that the configuration space $C(G-e, p, q)$ is bounded. The fact that $G$ is rigid in $\mathbb{R}^{d}$ implies that each connected component $H$ of $G-e$ with $n \geq 2$ vertices contains at least $d$ loops. (This follows since the dimension of the kernel of $R(G-e, p, q)$ is one, so the dimension of the kernel of $R\left(H,\left.p\right|_{H},\left.q\right|_{H}\right)$ is at most one. This implies that the rank of $R\left(H,\left.p\right|_{H},\left.q\right|_{H}\right)$ is at least $d n-1$. On the other hand, the rank of the submatrix of $R\left(H,\left.p\right|_{H},\left.q\right|_{H}\right)$ consisting of the rows indexed by the (non-loop) edges of $H$ is at most $d n-\left(\begin{array}{c}d+1 \\ 2\end{array}\right)$ when $n \geq d$ and $\left(\begin{array}{l}n \\ 2\end{array}\right)$ when $n<d$. This implies that the number of loops in $H$ is at least $(d n-1)-d n+\left(\begin{array}{c}d+1 \\ 2\end{array}\right)$ when $n \geq d$, and at least $(d n-1)-\left(\begin{array}{l}n \\ 2\end{array}\right)$ when $n<d$.)

It remains to show that each connected component $H$ of $G-e$ with exactly one vertex $v$, has at least $d$ loops. Suppose not. The facts that $G$ is rigid and connected imply that $H$ has exactly $(d-1)$ loops and that $e=u v$ for some $u \neq v$. Let $\ell$ be the line through $p(v)$ 
which is perpendicular to $q(f)$ for all loops incident to $v$ and let $P$ be the point on $\ell$ which is closest to $p(u)$. Let $p^{\prime}(x)=p(x)$ for all $x \in V-v$ and $p^{\prime}(v)=2 P-p(v)$. Then $\left(G, p^{\prime}, q\right)$ is equivalent to $(G, p, q)$ and $p^{\prime} \neq p$. This contradicts the fact that $(G, p, q)$ is globally rigid. Hence $C(G-e, p, q)$ is bounded.

We can now use a similar argument to that given in [16] to deduce that the component $\mathcal{C}$ of $C(G-e, p, q)$ that contains $p$ is diffeomorphic to a circle, and that there exists a $p^{\prime} \in \mathcal{C}-p$ with $\left(G, p^{\prime}, q\right)$ equivalent to $(G, p, q)$.

We will show in Section 8 that the necessary conditions for generic global rigidity given in Theorem 3.2 are also sufficient when $d=2$.

\section{Equilibrium STRESSES}

We will obtain an algebraic sufficient condition for a generic linearly constrained framework in $\mathbb{R}^{d}$ to be globally rigid, and show that the property that this condition holds is preserved by the graph '1-extension operation'. These results are key to our characterisation of global rigidity for 2-dimensional generic frameworks.

An equilibrium stress for a linearly constrained framework $(G, p, q)$ in $\mathbb{R}^{d}$ is a pair $(\omega, \lambda)$, where $\omega: E \rightarrow \mathbb{R}, \lambda: L \rightarrow \mathbb{R}$ and $(\omega, \lambda)$ belongs to the cokernel of $R(G, p, q)$. Thus $(\omega, \lambda)$ is an equilibrium stress for $(G, p)$ in $\mathbb{R}^{d}$ if and only if, for all $v_{i} \in V$,

$$
\sum_{v_{j} \in V} \omega_{i j}\left(p\left(v_{i}\right)-p\left(v_{j}\right)\right)+\sum_{e_{j} \in L} \lambda_{i, j} q\left(e_{j}\right)=0 .
$$

where $\omega_{i j}$ is taken to be equal to $\omega(e)$ if $e=v_{i} v_{j} \in E$ and to be equal to 0 if $v_{i} v_{j} \notin E$, and $\lambda_{i j}$ is equal to $\lambda\left(e_{j}\right)$ if $e_{j}$ is a loop at $v_{i}$ and is equal to 0 otherwise. An example is presented in Figure 2 ,

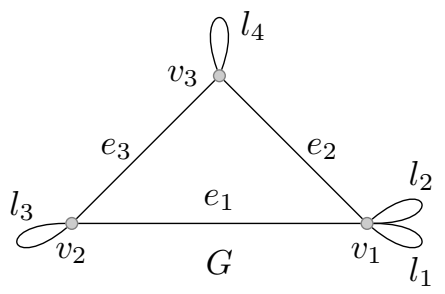

$$
\begin{aligned}
& p\left(v_{1}\right)=(1,0) \\
& p\left(v_{2}\right)=(-1,0) \\
& p\left(v_{3}\right)=(0,1) \\
& q\left(l_{1}\right)=(1,-1) \\
& q\left(l_{2}\right)=(1,0) \\
& q\left(l_{3}\right)=(1,1) \\
& q\left(l_{4}\right)=(0,1)
\end{aligned}
$$

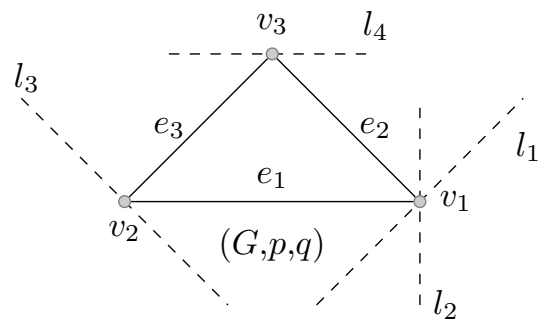

Figure 2. A looped simple graph $G$ and the corresponding realisation as a linearly constrained framework in $\mathbb{R}^{2}$. The vectors $\omega=(0,1,1)$ and $\lambda=$ $(-1,0,1,-2)$ give an equilibrium stress for this framework.

We can write (4.1) in matrix form

$$
\Pi(p) \Omega(\omega)+\Pi(q) \Lambda(\lambda)=0 .
$$

where:

- the stress matrix $\Omega(\omega)$ is a $|V| \times|V|$-matrix in which the off diagonal entry in row $v_{i}$ and column $v_{j}$ is $-\omega_{i j}$, and the diagonal entry in row $v_{i}$ is $\sum_{v_{j} \in V} \omega_{i j}$;

- the linear constraint matrix $\Lambda(\lambda)$ is a $|V| \times|L|$-matrix in which the entry in row $v_{i}$ and column $e_{j}$ is $\lambda_{i j}$;

- for any set $S=\left\{s_{1}, s_{2}, \ldots, s_{t}\right\}$ and map $f: S \rightarrow \mathbb{R}^{d}$, the coordinate matrix $\Pi(f)$ is the $d \times t$ matrix in which the $i$ 'th column is $f\left(s_{i}\right)$. 
Lemma 4.1. Suppose $(\omega, \lambda)$ is an equilibrium stress for a d-dimensional linearly constrained framework $(G, p, q)$. Then $\operatorname{rank} \Omega(\omega) \leq|V|-1$. In addition, if $(G, p)$ is generic and $G$ has at least two vertices and at most $d-1$ loops, then $\operatorname{rank} \Omega(\omega) \leq|V|-2$.

Proof. The first assertion follows from the fact that the vector $(1,1, \ldots, 1)$ belongs to the cokernel of $\Omega(\omega)$. To prove the second assertion, we choose a nonzero vector $q_{0} \in \mathbb{R}^{d}$ such that $q_{0}$ is orthogonal to $q(f)$ for all loops $f$ of $G$. Then $\left(p\left(v_{1}\right) \cdot q_{0}, p\left(v_{2}\right) \cdot q_{0}, \ldots, p\left(v_{n}\right)\right.$. $q_{0}$ ) belongs to the cokernel of $\Omega(\omega)$ by Equation (4.1), and is linearly independent from $(1,1, \ldots, 1)$ since $(G, p, q)$ is generic.

We say that $(\omega, \lambda)$ is a full rank equilibrium stress for $(G, p, q)$ if $\operatorname{rank} \Omega(\omega)=|V|-1$. For example, the framework $(G, p, q)$ drawn in Figure 2 has a stress matrix

$$
\Omega(\omega)=\left[\begin{array}{rrr}
1 & 0 & -1 \\
0 & 1 & -1 \\
-1 & -1 & 2
\end{array}\right]
$$

with respect to the given equilibrium stress $(\omega, \lambda)=(0,1,1,-1,0,1,-2)$ and we have $\operatorname{rank} \Omega(\omega)=2$. Thus $(\omega, \lambda)$ is a full rank equilibrium stress for the framework $(G, p, q)$ drawn in Figure 2, We will show in Theorem 4.5 below that the property of having a full rank equilibrium stress is a sufficient condition for a generic linearly constrained framework to be globally rigid in $\mathbb{R}^{d}$.

Lemma 4.2. Let $(G, p, q)$ and $\left(G, p^{\prime}, q\right)$ be frameworks and $(\omega, \lambda)$ be a full rank equilibrium stress for both $(G, p, q)$ and $\left(G, p^{\prime}, q\right)$. Then, for some fixed $t \in \mathbb{R}^{d}$, we have $p\left(v_{i}\right)=t+p^{\prime}\left(v_{i}\right)$ for all $v_{i} \in V(G)$.

Proof. Since $\operatorname{rank} \Omega(\omega)=|V(G)|-1$, the cokernel of $\Omega(\omega)$ is spanned by $(1,1, \ldots, 1)$. Since $(\omega, \lambda)$ is an equilibrium stress for both $(G, p, q)$ and $\left(G, p^{\prime}, q\right)$, we may use (4.2) to deduce that $\left(\Pi(p)-\Pi\left(p^{\prime}\right)\right) \Omega(\omega)=0$. This implies that each row of $\Pi\left(p-p^{\prime}\right)$ belongs to coker $\Omega(\omega)$ and hence is a scalar multiple of $(1,1, \ldots, 1)$. This gives $p\left(v_{i}\right)=t+p^{\prime}\left(v_{i}\right)$ for all $v_{i} \in V$, where $t=\left(t_{1}, t_{2}, \ldots, t_{d}\right)$ and $t_{i}(1,1, \ldots, 1)$ is the $i$ 'th row of $\Pi\left(p-p^{\prime}\right)$.

Lemma 4.3. Let $(G, p, q)$ be a generic linearly constrained framework in $\mathbb{R}^{d}$ and $\left(G, p^{\prime}, q\right)$ be an equivalent framework. Suppose that $G$ has at least $d$ loops and that $p(v)=p^{\prime}(v)+t$ for some fixed $t \in \mathbb{R}^{d}$, for all $v \in V$. Then $p=p^{\prime}$.

Proof. Choose distinct loops $e_{i} \in L$ for $1 \leq i \leq d$ and let $v_{i}$ be the vertex incident to $e_{i}$. Let $P, P^{\prime}$ and $Q$ be the $d \times d$ matrices whose $i$ 'th columns are $p\left(v_{i}\right), p^{\prime}\left(v_{i}\right)$ and $q\left(e_{i}\right)$, respectively and let $A$ be the $d \times d$ diagonal matrix with the coordinates of $t$ on the diagonal. Then $\left(P-P^{\prime}\right) Q^{T}=0$ since $(G, p, q)$ and $\left(G, p^{\prime}, q\right)$ are equivalent. Since $P-P^{\prime}=A J$ where $J$ is the $d \times d$ matrix all of whose entries are 1 , we have $A J Q^{T}=0$. Since $(G, p, q)$ is generic, $Q$ is nonsingular and hence $A J=0$. This implies that $A=0$ and hence $t=0$.

Proposition 4.4 (Connelly [5]). Suppose that $f: \mathbb{R}^{a} \rightarrow \mathbb{R}^{b}$ is a function, where each coordinate is a polynomial with coefficients in some finite extension $\mathbb{K}$ of $\mathbb{Q}, p \in \mathbb{R}^{a}$ is generic over $\mathbb{K}$ and $f(p)=f(\hat{p})$, for some $\hat{p} \in \mathbb{R}^{a}$. Then there are (open) neighbourhoods $N_{p}$ of $p$ and $N_{\hat{p}}$ of $\hat{p}$ in $\mathbb{R}^{a}$ and a diffeomorphism $g: N_{\hat{p}} \rightarrow N_{p}$ such that for all $x \in N_{q}$, $f(g(x))=f(x)$, and $g(\hat{p})=p$.

Theorem 4.5. Suppose $(G, p, q)$ is a generic linearly constrained framework in $\mathbb{R}^{d}$ with at least two vertices, and $(\omega, \lambda)$ is a full rank equilibrium stress for $(G, p, q)$. Then $(G, p, q)$ is globally rigid. 
Proof. Let $(G, \hat{p}, q)$ be equivalent to $(G, p, q)$. Let $F: \mathbb{R}^{d|V|} \rightarrow \mathbb{R}^{|E|+|L|}$ be the rigidity map defined by $F(p)=\left(F_{E}(p), F_{L}(p)\right)$ where $F_{E}: \mathbb{R}^{d|V|} \rightarrow \mathbb{R}^{|E|}$ is the usual rigidity map defined by $F_{E}(p)=(\ldots,\|p(u)-p(v)\|, \ldots)_{e=u v \in E}$, and $F_{L}: \mathbb{R}^{d|V|} \rightarrow \mathbb{R}^{|L|}$ is defined by $F_{L}(p)=(\ldots, p(v) \cdot q(f), \ldots)_{f=v v \in L}$.

Proposition 4.4 implies that there exist neighbourhoods $N_{p}$ of $(p, q)$ and $N_{\hat{p}}$ of $(\hat{p}, q)$ and a diffeomorphism $g: N_{\hat{p}} \rightarrow N_{p}$ such that $g(\hat{p})=p$ and for all $\bar{p} \in N_{\hat{p}}$ we have $F(g(\hat{p}))=F(\hat{p})$. By differentiating at $\hat{p}$, observing that the differential of $F$ is (up to scaling) the rigidity matrix $R(G, p, q)$ and using the fact that $(\omega, \lambda)$ is an equilibrium stress for $(G, p, q)$, we have $(\omega, \lambda) R(G, \hat{p}, q)=(\omega, \lambda) R(G, p, q) D=0 \cdot D=0$ where $D$ is the Jacobean of $g$ at $\hat{p}$. Hence $(\omega, \lambda)$ is an equilibrium stress for $(G, \hat{p}, q)$. We can now use Lemmas Lemma 4.1, 4.2 and 4.3 to deduce that $p=\hat{p}$.

We will prove a partial converse to Theorem 4.5 in Section 8 by showing that every connected, 2-dimensional, globally rigid generic framework with at least two vertices has a full rank equilibrium stress. Note that the converse to Theorem 4.5 is false for disconnected frameworks since a framework is globally rigid if and only if each of its connected components is globally rigid, whereas no disconnected framework can have a full rank equilibrium stress (since we may apply Lemma 4.1 to each connected component).

Our next result shows that we can apply Theorem 4.5 whenever we can find a full rank equilibrium stress in an arbitrary infinitesimally rigid framework.

Lemma 4.6. Suppose $G=(V, E, L)$ can be realised in $\mathbb{R}^{d}$ as an infinitesimally rigid linearly constrained framework with a full rank equilibrium stress. Then every generic realisation of $G$ in $\mathbb{R}^{d}$ is infinitesimally rigid and has a full rank equilibrium stress which is nonzero on all elements of $E \cup L$.

Proof. Let $|V|=n,|E \cup L|=m$, and let $(G, p, q)$ be a realisation of $G$ in $\mathbb{R}^{d}$. Since the entries in the rigidity matrix $R(G, p, q)$ are polynomials in $p$ and $q$, the rank of $R(G, p, q)$ will be maximised whenever $\left(G, p^{\prime}, q^{\prime}\right)$ is generic. Hence $(G, p, q)$ will be infinitesimally rigid whenever $(G, p, q)$ is generic.

We adapt the proof technique of Connelly and Whiteley [6, Theorem 5] to prove the second part of the theorem. Since the entries in $R(G, p, q)$ are polynomials in $p$ and $q$, and the space of equilibrium stresses of $(G, p, q)$ is the cokernel of $R(G, p, q)$, each equilibrium stress of $(G, p, q)$ can be expressed as a pair of rational functions $(\omega(p, q, t), \lambda(p, q, t))$ of $p$, $q$ and $t$, where $t$ is a vector of $m-d n$ indeterminates. This implies that the entries in the corresponding stress matrix $\Omega(\omega(p, q, t))$ will also be rational functions of $p, q$ and $t$. Hence the rank of $\Omega(\omega(p, q, t))$ will be maximised whenever $p, q, t$ is algebraically independent over $\mathbb{Q}$. In particular, for any generic $p, q \in \mathbb{R}^{d n}$, we can choose $t \in \mathbb{R}^{m-d n}$ such that $\operatorname{rank} \Omega(\omega(p, q, t))=d n-1$ and hence $(\omega(p, q, t), \lambda(p, q, t))$ will be a full rank equilibrium stress for $(G, p, q)$.

Now suppose that $(G, p, q)$ is generic and that $(\omega, \lambda)$ is a full rank stress for $(G, p, q)$ chosen such that the total number of edges $e \in E \cup L$ with $\omega_{e}=0$ and loops $\ell \in L$ with $\lambda_{\ell}=0$ is as small as possible. We may assume that, for some $f \in E \cup L$, we have $\omega_{f}=0$ if $f \in E$ and $\lambda_{f}=0$ if $f \in L$. Then $\left(\left.\omega\right|_{E-f},\left.\lambda\right|_{L-f}\right)$ is a full rank equilibrium stress for $(G-f, p, q)$. By Theorem 4.5, $\left(G-f, p,\left.q\right|_{L-f}\right)$ is globally rigid. In particular $\left(G-f, p,\left.q\right|_{L-f}\right)$ is rigid, and hence, since $\left(G-f, p,\left.q\right|_{L-f}\right)$ is generic, it is infinitesimally rigid. This implies that the row of $R(G, p, q)$ indexed by $f$ is contained in a minimal linearly dependent set of rows, and gives us an equilibrium stress $(\hat{\omega}, \hat{\lambda})$ for $(G, p, q)$ with $\hat{\omega}_{f} \neq 0$ when $f \in E$, and $\hat{\lambda}_{f} \neq 0$ when $f \in L$. Then $\left(\omega^{\prime}, \lambda^{\prime}\right)=(\omega, \lambda)+c(\hat{\omega}, \hat{\lambda})$ is an equilibrium stress for $(G, p)$, for any $c \in \mathbb{R}$. We can now choose a sufficiently small $c>0$ so that $\operatorname{rank} \Omega\left(\omega^{\prime}\right)=n-1, \omega_{e}^{\prime} \neq 0$ 
for all $e \in E$ for which $\omega_{e} \neq 0$, and $\lambda_{\ell}^{\prime} \neq 0$ for all $\ell \in L$ for which $\lambda_{\ell} \neq 0$. This contradicts the choice of $(\omega, \lambda)$.

Theorem 4.5. Lemma 4.6 and the fact that the framework $(G, p, q)$ drawn in Figure 2 is infinitesimally rigid and has a full rank equilibrium stress imply that every generic realisation of $G$ as a linearly constrained framework in $\mathbb{R}^{2}$ is globally rigid. A similar argument will allow us to show that the '1-extension operation' preserves the property of having a full rank equilibrium stress.

Let $G=(V, E, L)$ be a looped simple graph. The $d$-dimensional 1-extension operation forms a new looped simple graph from $G$ by deleting an edge or loop $e \in E \cup L$ and adding a new vertex $v$ and $d+1$ new edges or loops incident to $v$, with the provisos that each end vertex of $e$ is incident to exactly one new edge, and, if $e \in L$, then there is at least one new loop incident to $v$. See Figure 3 for an illustration of the types of 1-extension we will use.
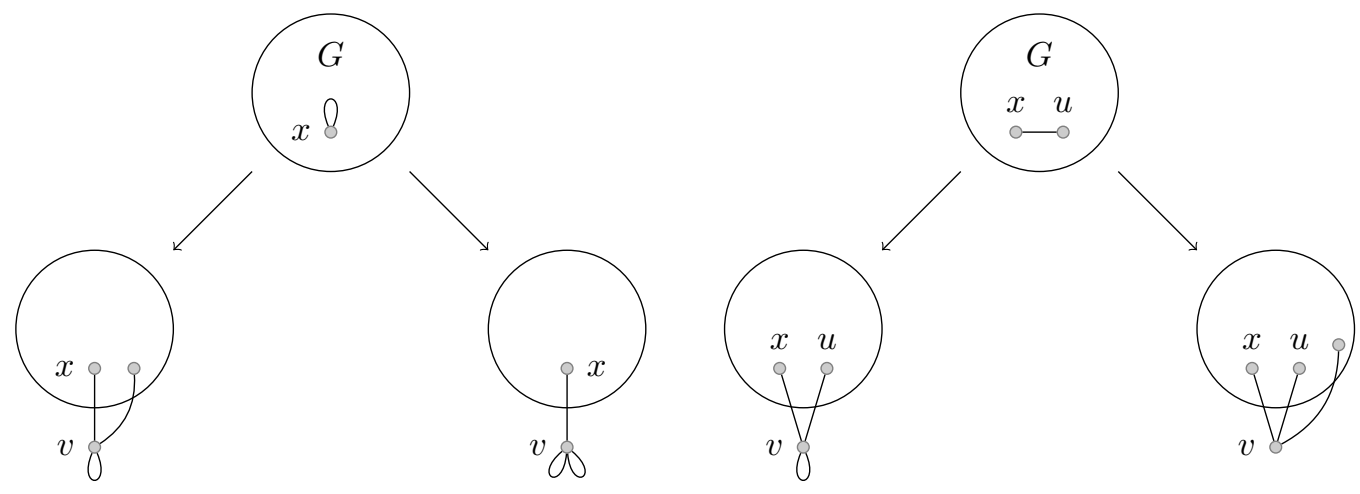

Figure 3. Possible 2-dimensional 1-extensions on a loop (on the left) and on an edge (on the right) of a graph $G$.

Theorem 4.7. Let $H$ be a looped simple graph and $G$ be obtained from $H$ by either adding an edge or a loop, or by applying the d-dimensional 1-extension operation. Suppose that $H$ can be realised in $\mathbb{R}^{d}$ as an infinitesimally rigid linearly constrained framework with a full rank equilibrium stress. Then $G$ can be realised in $\mathbb{R}^{d}$ as an infinitesimally rigid linearly constrained framework with a full rank equilibrium stress.

Proof. Let $(H, p, q)$ be a generic realisation of $H$ as a $d$-dimensional linearly constrained framework. By Lemma 4.6. $(H, p, q)$ has a full rank equilibrium stress $(\omega, \lambda)$ which is nonzero on all elements of $E \cup L$.

Suppose $G$ is obtained from $H$ by adding an edge or loop $f$. Let $\left(G, p, q^{\prime}\right)$ be the realisation of $G$ obtained from $(H, p, q)$ by assigning an arbitrary value to $q^{\prime}(f)$ if $f$ is a loop. Then $\left(G, p, q^{\prime}\right)$ is infinitesimally rigid and the equilibrium stress $\left(\omega^{\prime}, \lambda^{\prime}\right)$ for $\left(G, p, q^{\prime}\right)$ obtained from $(\omega, \lambda)$ by setting $\left(\omega^{\prime}, \lambda^{\prime}\right)$ to be zero on $f$ has full rank. Hence we may suppose that $G$ is a 1-extension of $H$.

There are two cases to consider depending on whether the 1-extension deletes an edge or a loop. In the former case we can proceed by the standard collinear triangle technique given in [5] (if a new edge is a loop then the loop is assigned stress 0). Hence we present the latter case.

Let $V=\left\{v_{1}, v_{2}, \ldots, v_{n}\right\}$. Suppose that the 1-extension deletes $f_{1} \in L$, where $f_{1}$ is a loop at $v_{1}$ and adds a new vertex $v_{0}$ with neighbours $v_{1}, v_{2}, \ldots, v_{k_{1}}$ and loops $f_{0}^{1}, \ldots, f_{0}^{k_{2}}$ at $v_{0}$ 
(where $k_{1}+k_{2}=d+1$ ). Let $\left(G, p^{\prime}, q^{\prime}\right)$ be defined by putting $p^{\prime}(v)=p(v)$ for all $v \in V$, $q^{\prime}(f)=q(f)$ for all $f \in L-f_{1}, p^{\prime}\left(v_{0}\right)=p\left(v_{1}\right)+q\left(f_{1}\right)$ and $q^{\prime}\left(f_{0}^{1}\right)=q\left(f_{1}\right)$ and choosing $\left\{q\left(f_{0}^{1}\right), \ldots, q\left(f_{0}^{k_{2}}\right)\right\}$ to be algebraically independent.

We first show that the framework $\left(G+f_{1}-v_{0} v_{1}, p^{\prime}, q^{\prime}\right)$ is infinitesimally rigid. Its rigidity matrix $R$ can be constructed from $R(H, p, q)$ by adding $d$ new columns indexed by $v_{0}$, and $d$ new rows indexed by $v_{0} v_{2}, \ldots v_{0} v_{k_{1}}, f_{0}^{1}, \ldots, f_{0}^{k_{2}}$, respectively. Since $(p, q)$ is generic the $d \times d$ submatrix $M$ of $R$ with rows indexed by $v_{0} v_{2}, \ldots v_{0} v_{k_{1}}, f_{0}^{1}, \ldots, f_{0}^{k_{2}}$ and columns indexed by $v_{0}$ is nonsingular. The fact that the new columns contain zeros everywhere except in the new rows now gives $\operatorname{rank} R=\operatorname{rank} R(H, p, q)+d$. By the choice of $p^{\prime}, q^{\prime}$, the rows in $R$ corresponding to $v_{0} v_{1}, f_{1}, f_{0}^{1}$ are a minimal linearly dependent set. Thus

$$
\operatorname{rank} R\left(G, p^{\prime}, q^{\prime}\right)=\operatorname{rank} R=\operatorname{rank} R(H, p, q)+d .
$$

The fact that $(H, p, q)$ is infinitesimally rigid now implies that $\left(G, p^{\prime}, q^{\prime}\right)$ is infinitesimally rigid.

Let $\left(\omega^{\prime}, \lambda^{\prime}\right)$ be the stress for $\left(G, p^{\prime}, q^{\prime}\right)$ defined by putting $\omega_{e}^{\prime}=\omega_{e}$ for all $e \in E, \omega_{v_{0} v_{i}}^{\prime}=0$ for all $i>1, \omega_{v_{0} v_{1}}^{\prime}=-\lambda\left(f_{1}\right), \lambda^{\prime}(f)=\lambda(f)$ for all $f \in L, \lambda^{\prime}\left(f_{0}^{1}\right)=\lambda\left(f_{1}\right)$ and $\lambda^{\prime}\left(f_{0}^{i}\right)=0$ for all $i>1$. It is straightforward to verify that $\left(\omega^{\prime}, \lambda^{\prime}\right)$ is an equilibrium stress for $\left(G, p^{\prime}, q^{\prime}\right)$. We let $\omega_{i j}$ denote the stress $\omega_{e}^{\prime}=\omega_{e}$ on $e=v_{i} v_{j} \in E$ and put $\lambda_{1}=\lambda\left(f_{1}\right)$. We have

$$
\Omega\left(\omega^{\prime}\right)=\left[\begin{array}{cccccc}
-\lambda_{1} & \lambda_{1} & 0 & 0 & \ldots & 0 \\
\lambda_{1} & \sum_{j \geq 1} \omega_{1 j}-\lambda_{1} & -\omega_{12} & -\omega_{13} & \ldots & -\omega_{1 n} \\
0 & -\omega_{12} & \sum_{j \geq 1} \omega_{2 j} & -\omega_{23} & \ldots & -\omega_{2 n} \\
0 & -\omega_{13} & -\omega_{23} & \sum_{j \geq 1} \omega_{3 j} & \ldots & -\omega_{3 n} \\
\vdots & \vdots & \vdots & & & \vdots
\end{array}\right] .
$$

By adding row 1 to row 2 and then column 1 to column 2 this reduces to

$$
\left[\begin{array}{cccccc}
-\lambda_{1} & 0 & 0 & 0 & \ldots & 0 \\
0 & \sum_{j} \omega_{1 j} & -\omega_{12} & -\omega_{13} & \ldots & -\omega_{1 n} \\
0 & -\omega_{12} & \sum_{j} \omega_{2 j} & -\omega_{23} & \ldots & -\omega_{2 n} \\
\vdots & \vdots & \vdots & \vdots & & \vdots
\end{array}\right]=\left[\begin{array}{cccc}
-\lambda_{1} & 0 & \ldots & 0 \\
0 & & & \\
\vdots & \Omega(\omega) & \\
0 & & &
\end{array}\right] .
$$

Since $-\lambda_{1} \neq 0$, we have $\operatorname{rank} \Omega\left(\omega^{\prime}\right)=\operatorname{rank} \Omega(\omega)+1$. Hence $\left(\omega^{\prime}, \lambda^{\prime}\right)$ is a full rank equilibrium stress for $\left(G, p^{\prime}, q^{\prime}\right)$.

\section{Circuits in the 2-Dimensional Linearly COnstrained Rigidity matroid}

We will focus on 2-dimensional linearly constrained frameworks in the following sections, and will suppress specific reference to the dimension. In particular we will refer to the 2-dimensional 1-extension operation as a 1-extension, to the property of being 2-balanced as balanced, and say that a graph is rigid to mean it is rigid in $\mathbb{R}^{2}$.

This section will contain a combinatorial analysis of the simplest redundantly rigid graphs - these correspond to rigid circuits in the linearly constrained generic rigidity matroid. Our results are analogous to those obtained in 11 for bar-joint frameworks and [14 for directionlength frameworks.

Let $G=(V, E, L)$ be a looped graph. For $X \subset V$, we define a matroid $\mathcal{M}_{l c}(G)$ on $E \cup L$ by the conditions of Theorem 2.1, a set $F \subset E \cup L$ is independent if $\left|F^{\prime}\right| \leq 2\left|V_{F^{\prime}}\right|$ for all $F^{\prime} \subseteq F$, and $\left|F^{\prime}\right| \leq 2\left|V_{F^{\prime}}\right|-3$ for all $\emptyset \neq F^{\prime} \subseteq F \cap E$. We will refer to subgraphs of $G$ whose edge-set is a circuit in $\mathcal{M}_{l c}(G)$ as $\mathcal{M}_{l c}$-circuits, see Figure 4. The definition of independence in $\mathcal{M}_{l c}(G)$ gives rise to the following characterisation of its circuits. For $X \subseteq V$, let $i_{E}(X)$ 

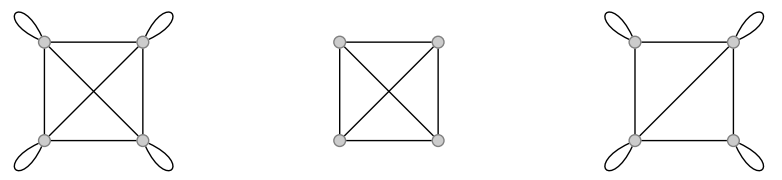

Figure 4. A graph $G$ on the left and two $\mathcal{M}_{l c}$-circuits of $G$ in the middle and on the right. It can be verified by Lemma 5.1 that the subgraph in the middle is a flexible $\mathcal{M}_{l c^{-}}$-circuit and the subgraph on the right is a rigid $\mathcal{M}_{l c^{-}}$ circuit.

and $i_{L}(X)$ denote the number of elements of $E$ and $L$ respectively in the subgraph induced by $X$ in $G$ and put $i_{E \cup L}(X)=i_{E}(X)+i_{L}(X)$.

Lemma 5.1. Let $G=(V, E, L)$ be a looped simple graph. Then $G$ is an $\mathcal{M}_{l c}$-circuit if and only if, either

(a) $|E|+|L|=2|V|+1, i_{E}(X) \leq 2|X|-3$ for all $X \subset V$ with $|X| \geq 2$ and $i_{E \cup L}(X) \leq 2|X|$ for all $X \subsetneq V$, or

(b) $L=\emptyset,|E|=2|V|-2$ and $i_{E}(X) \leq 2|X|-3$ for all $X \subsetneq V$ with $|X| \geq 2$.

We will refer to the circuits described in (a) as rigid $\mathcal{M}_{l c}$-circuits and to those in (b) as flexible $\mathcal{M}_{l c}$-circuits. The smallest rigid $\mathcal{M}_{l c}$-circuits are the looped graphs $K_{1}^{[3]}$ and $K_{2}^{[2]}$. The flexible $\mathcal{M}_{l c}$-circuits are precisely the circuits of the 2-dimensional generic bar-joint rigidity matroid. A recursive construction for these circuits is given in [1]. We will use the results of this section to obtain a recursive construction for rigid $\mathcal{M}_{l c}$-circuits in Section 6 ,

Let $d^{\dagger}(v)$ denote the number of edges or loops that are incident with a vertex $v$ in a looped simple graph $G, d(v)=d^{\dagger}(v)+i_{L}(v)$ denote the degree of $v$ in $G$, and $V_{3}=\{v \in$ $\left.V: d^{\dagger}(v)=3\right\}$ denote the set of nodes of $G$. For disjoint $X, Y \subseteq V$, let $G[X]$ denote the subgraph of $G$ induced by $X$ and $d(X, Y)$ denote the number of edges of $G$ between $X$ and $Y$.

Lemma 5.2. Let $G=(V, E, L)$ be a rigid $\mathcal{M}_{l c}$-circuit. Then $V_{3} \neq \emptyset$ and the subgraph $G^{\dagger}\left[V_{3}\right]$ of $G$ obtained by deleting all loops in $G\left[V_{3}\right]$ is a forest.

Proof. Since $G$ is a rigid $\mathcal{M}_{l c}$-circuit, $d^{\dagger}(v) \geq 3$ for all $v \in V$,

$$
4|V|+2=2|E|+2|L|=\sum_{v \in V} d(v)=\sum_{v \in V} d^{\dagger}(v)+|L|,
$$

and $|L| \geq 3$. This gives $\sum_{v \in V} d^{\dagger}(v) \leq 4|V|-1$ so $G$ has at least one node.

Suppose $C=(S, F)$ is an induced cycle in $G^{\dagger}\left[V_{3}\right]$. Let $T=V \backslash S$. Then we have

$$
\begin{aligned}
i_{E \cup L}(T) & =2|V|+1-i_{E \cup L}(S)-d(S, T) \\
& =2|V|+1-|S|-i_{L}(S)-d(S, T) \\
& =2|T|+1,
\end{aligned}
$$

where the second equality follows from the fact that $C$ is a cycle, and the last equality follows from the fact that a vertex in $S$ contributes 1 to exactly one of $i_{L}(S)$ and $d(S, T)$ so $i_{L}(S)+d(S, T)=|S|$. This contradicts the fact that $G$ is a rigid $\mathcal{M}_{l c}$-circuit and $T$ is a proper subset of $V$.

Let $G=(V, E, L)$ be a rigid $\mathcal{M}_{l c}$-circuit. We say that $X \subseteq V$ is mixed critical if $i_{E \cup L}(X)=2|X|$ and is pure critical if $i_{E}(X)=2|X|-3$. We say that $X$ is critical if it is either mixed or pure critical. 
Lemma 5.3. Let $X$ be a mixed critical set in a rigid $\mathcal{M}_{l c}$-circuit $G=(V, E, L)$ and $Y=$ $V \backslash X$.

(a) Then $\left|V_{3} \cap Y\right| \geq 1$, with strict inequality when $|Y| \geq 2$.

(b) If $d(X, Y) \geq 3$ then $Y$ contains a vertex of degree three in $G$.

Proof. (a) If $|Y|=1$ then the unique vertex in $Y$ must be a node of $G$. Hence we may assume that $|Y| \geq 2$.

Suppose $\left|Y \cap V_{3}\right| \leq 1$. Note that for a vertex $v \in Y$, we have $d(v)=d^{\dagger}(v)+i_{L}(v)$ so

$$
\sum_{v \in Y} d(v)=\sum_{v \in Y} d^{\dagger}(v)+i_{L}(Y) \geq 4|Y|-1+i_{L}(Y)
$$

We also have

$$
2 i_{E \cup L}(Y)=\sum_{v \in Y} d_{G[Y]}(v)=\sum_{v \in Y} d(v)-d(Y, X) .
$$

We can combine these two (in)equalities to obtain

$$
2 i_{E}(Y)+i_{L}(Y)+d(Y, X) \geq 4|Y|-1 .
$$

Since $|Y| \geq 2$ we have $i_{E}(Y) \leq 2|Y|-3$ and Equation (5.1) now gives

$$
i_{E \cup L}(Y)+d(Y, X)=i_{E}(Y)+i_{L}(Y)+d(Y, X) \geq 4|Y|-1-i_{E}(Y) \geq 2|Y|+2 .
$$

The fact that $X$ is mixed critical now gives

$$
|E|+|L|=i_{E \cup L}(X)+i_{E \cup L}(Y)+d(Y, X) \geq 2|X|+2|Y|+2=2|V|+2,
$$

a contradiction.

(b) We have $\sum_{v \in Y} d(v)=2 i_{E \cup L}(Y)+d(Y, X)$ and

$$
i_{E \cup L}(Y)=|E|-i_{E \cup L}(X)-d(Y, X)=2|V \backslash X|-d(Y, X)+1 .
$$

This implies that

$$
\sum_{v \in Y} d(v)=4|V \backslash X|-2 d(Y, X)+d(Y, X)+2=4|Y|-d(Y, X)+2 .
$$

If $d(Y, X) \geq 3$ then $\sum_{v \in Y} d(v)<4|Y|$ and hence $Y$ contains a vertex of degree less than four.

We next consider two versions of the 1-extension operation for a looped simple graph $G=(V, E, L)$. The 1-extension operation at an edge $u w \in E$ deletes $u w$ and adds a new vertex $v$, new edges $u v$ and $w v$ and either a new edge $v x$ for some $x \in V \backslash\{u, w\}$ or a new loop at $v$. The 1-extension operation at a loop $u u \in L$ deletes $u u$, adds a new vertex $v$, a new edge $u v$ and a new loop at $v$, and either a new edge $w v$, for some $w \in V-u$ or a second new loop at $v$. It is straightforward to show that both versions of the 1-extension operation transform a rigid $\mathcal{M}_{l c}$-circuit into another rigid $\mathcal{M}_{l c}$-circuit using Lemma 5.1(a).

We refer to the inverse operation to each of the above 1-extension operations as a 1reduction to an edge or loop, respectively. When $G$ is a rigid $\mathcal{M}_{l c}$-circuit and $v \in V$, we say that these reduction operations are admissible if they result in a smaller rigid $\mathcal{M}_{l c}$-circuit, and that $v$ is admissible if there is an admissible 1-reduction at $v$.

The remainder of this section will be devoted to obtaining a structural characterisation of 'non-admissibility'. This will be used in the next section to show that every balanced rigid $\mathcal{M}_{l c}$-circuit on at least two vertices contains an admissible vertex. 
Lemma 5.4. Let $G=(V, E, L)$ be a rigid $\mathcal{M}_{l c}$-circuit and $v$ be a node in $G$.

(a) Suppose $N(v)=\{u, w, z\}$. Then the 1-reduction at $v$ which adds uw is non-admissible if and only if there exists a pure critical set $X$ with $u, w \in X$ and $v, z \notin X$ or a mixed critical set $Y$ with $u, w \in Y$ and $v, z \notin Y$.

(b) Suppose $N(v)=\{u, w\}$. Then:

(i) the 1-reduction at $v$ which adds uw is non-admissible if and only if there exists a pure critical set $X$ with $u, w \in X$ and $v \notin X$;

(ii) the 1-reduction at $v$ which adds uu is non-admissible if and only if there exists a mixed critical set $X$ with $u \in X$ and $w, v \notin X$.

(c) If $N(v)=\{u\}$, then the 1-reduction at $v$ which adds uu is admissible.

Proof. It is straightforward to show that the existence of each of the critical sets described in (a) and (b) implies non-admissibility.

For the converse, we first suppose that the 1-reduction described in case (a) is nonadmissible. Then the graph resulting from this 1-reduction is not a rigid $\mathcal{M}_{l c}$-circuit. This implies that there exists either an $X \subset V-v$ with $i_{E}(X) \geq 2|X|-2$ or a $Y \subsetneq V-v$ with $i_{E \cup L}(Y) \geq 2|Y|+1$. These subsets are pure critical and mixed critical, respectively, in $G$. If the first alternative holds then $z \notin X$, since otherwise $i_{E}(X+v)=i_{E}(X)+3=2|X|-3+3=$ $2|X+v|-2$ and we would contradict the fact that $G$ is a rigid $\mathcal{M}_{l c}$-circuit. Similarly, if the second alternative holds and $z \in Y$ then $i_{E \cup L}(Y+v)=i_{E \cup L}(Y)+3=2|Y|+3=2|Y+v|+1$, again contradicting the fact that $G$ is a rigid $\mathcal{M}_{l c}$-circuit.

The arguments in cases (b) and (c) are similar.

Lemma 5.5. Let $G$ be a rigid $\mathcal{M}_{l c}$-circuit.

(a) If $X, Y \subset V$ are pure critical with $|X \cap Y| \geq 2$, then $X \cup Y$ and $X \cap Y$ are pure critical and $d(X \backslash Y, Y \backslash X)=0$.

(b) If $X, Y \subset V$ are mixed critical and $|X \cup Y| \leq|V|-1$, then $X \cup Y$ and $X \cap Y$ are mixed critical and $d(X \backslash Y, Y \backslash X)=0$.

(c) If $X \subset V$ is mixed critical, $Y \subset V$ is pure critical, $|X \cap Y| \geq 2$ and $|X \cup Y| \leq|V|-1$, then $X \cup Y$ is mixed critical, $X \cap Y$ is pure critical and $i_{L}(Y \backslash X)=0=d(X \backslash Y, Y \backslash X)$.

Proof. We prove (c), parts (a) and (b) can be proved similarly. We have

$$
\begin{aligned}
2|X|+2|Y|-3 & =i_{E \cup L}(X)+i_{E}(Y) \\
& =i_{E \cup L}(X \cup Y)+i_{E}(X \cap Y)-i_{L}(Y \backslash X)-d(X \backslash Y, Y \backslash X) \\
& \leq 2|X \cup Y|+2|X \cap Y|-3-i_{L}(Y \backslash X)-d(X \backslash Y, Y \backslash X) \\
& =2|X|+2|Y|-3-i_{L}(Y \backslash X)-d(X \backslash Y, Y \backslash X)
\end{aligned}
$$

Hence $i_{L}(Y \backslash X)=d(X-Y, Y-X)=0$ and equality holds throughout the above displayed calculation. In particular, $i_{E \cup L}(X \cup Y)=2|X \cup Y|, i_{E}(X \cap Y)=2|X \cap Y|-3$.

Lemma 5.6. Let $G$ be a rigid $\mathcal{M}_{l c}$-circuit and $v$ be a node of $G$ with three distinct neighbours $u, w, t$. Suppose that $X, Y$ are mixed critical sets in $G$ satisfying $\{u, w\} \subseteq X \subseteq V \backslash\{v, t\}$ and $\{w, t\} \subseteq Y \subseteq V \backslash\{v, u\}$. Suppose further that $Z$ is a (mixed or pure) critical set with $\{u, t\} \subseteq Z \subseteq V \backslash\{v, w\}$ Let $W^{\dagger}=(V-v) \backslash W$ for each $W \in\{X, Y, Z\}$. Then:

(a) $X \cup Y=X \cup Z=Y \cup Z=V-v$;

(b) $d\left(X^{\dagger}, Y^{\dagger}\right)=d\left(Y^{\dagger}, Z^{\dagger}\right)=d\left(X^{\dagger}, Z^{\dagger}\right)=0$;

(c) either $\left\{X^{\dagger}, Y^{\dagger}, Z^{\dagger}, X \cap Y \cap Z\right\}$ is a partition of $V-v$, or $X \cap Y \cap Z=\emptyset$ and $\left\{X^{\dagger}, Y^{\dagger}, Z^{\dagger}\right\}$ is a partition of $V-v$;

(d) if $Z$ is pure critical then $i_{L}\left(X^{\dagger}\right)=0=i_{L}\left(Y^{\dagger}\right)$. 
Proof. Since $X, Y$ are mixed critical, $X \cup Y$ is mixed critical and $d(X \backslash Y, Y \backslash X)=0$ by Lemma 5.5(b). The first assertion gives $i_{E \cup L}(X \cup Y \cup\{v\})=2|X \cup Y \cup\{v\}|+1$. Since $G$ is an $\mathcal{M}_{l c}$-circuit, this imples that $X \cup Y=V-v$. We now have $X^{\dagger}=Y \backslash X$ and $Y^{\dagger}=X \backslash Y$. When $Z$ is mixed critical, a similar argument for $X, Z$ and $Y, Z$ tells us that (a) and (b) hold. Part (c) follows immediately from (a). Hence we may assume that $Z$ is pure critical.

Since $Z$ is pure critical, $G[Z]$ is connected and hence there is a path $P$ in $G[Z]$ from $u$ to $t$. If $X \cap Z=\{u\}$ then $P$ would contain no vertices of $X-u$. The existence of such a path $P$ would contradict the fact that $u \in X \backslash Y, t \in Y \backslash X$ and $d(X \backslash Y, Y \backslash X)=0$. Hence $|X \cap Z| \geq 2$ and we can use Lemma 5.5(c) to deduce that $X \cup Z$ is mixed critical and $d_{L}^{\dagger}\left(X^{\dagger}\right)=0=d(X \backslash Z, Z \backslash X)$. A similar argument as in the previous paragraph now gives $X \cup Z=V-v$. We can now use symmetry to deduce that $Y \cup Z=V-v$ and $d_{L}^{\dagger}\left(Y^{\dagger}\right)=0=d(Y \backslash Z, Z \backslash Y)$. This gives (a), (b), (c) and (d) in the case when $Z$ is pure critical.

We call a triple $(X, Y, Z)$ of three sets satisfying the hypotheses of Lemma 5.6 a strong flower on $v$ when $Z$ is mixed critical and a weak flower on $v$ when $Z$ is pure critical. Note that it is possible for $(X, Y, Z)$ to be both a strong and weak flower on $v$, see Figure 5 .

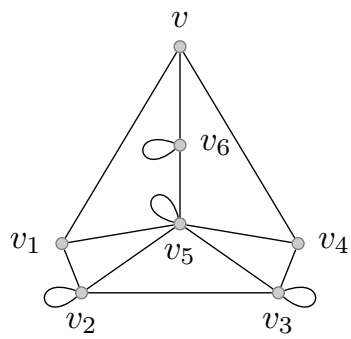

Figure 5. Let $X=\left\{v_{1}, v_{2}, v_{3}, v_{5}, v_{6}\right\}, Y=\left\{v_{2}, v_{3}, v_{4}, v_{5}, v_{6}\right\}$, and $Z=$ $\left\{v_{1}, v_{2}, v_{3}, v_{4}, v_{5}\right\}$. Then $Z$ is pure critical and mixed critical so $(X, Y, Z)$ is both a strong and a weak flower on $v$.

Lemma 5.7. Let $G$ be a rigid $\mathcal{M}_{l c}$-circuit and $v$ be a node of $G$ with three distinct neighbours $r, s, t$. Suppose that $Y, Z$ are pure critical sets satisfying $\{s, t\} \subseteq Y \subseteq V \backslash\{v, r\}$ and $\{r, s\} \subseteq Z \subseteq V \backslash\{v, t\}$, and $X$ is a critical set with $\{r, t\} \subseteq X \subseteq V \backslash\{v, s\}$. Then $X$ is mixed critical, $X \cap Y=\{t\}, X \cap Z=\{r\}, Y \cap Z=\{s\}, G-v=G[X] \cup G[Y] \cup G[Z]$, and the component of $G-\{r, t\}$ which contains $\{v, s\}$ has no loops.

Proof. If $|Y \cap Z| \geq 2$ then $Y \cup Z$ would be pure critical by Lemma 5.5(a) and we would have $i_{E}(Y \cup Z \cup\{v\})=2|Y \cup Z \cup\{v\}|-2$. This would contradict the fact that $G$ is a rigid $\mathcal{M}_{l c}$-circuit. Hence $Y \cap Z=\{s\}$. Since $Y$ is pure critical, $G[Y]$ is connected and hence there exists a path $P$ in $G$ from $s$ to $t$ which avoids $Z-s$.

Suppose $X$ is pure critical. Then a similar argument to the above gives $X \cap Z=\{r\}$ and $Y \cap X=\{t\}, X \cup Y \cup Z$ is pure critical and $i_{E}(X \cup Y \cup Z \cup\{v\})=2|X \cup Y \cup Z \cup\{v\}|-2$. This would again contradict the fact that $G$ is a rigid $\mathcal{M}_{l c}$-circuit. Hence $X$ is mixed critical.

Suppose $|X \cap Z|>1$. Then $X \cup Z$ is mixed critical and $d(X \backslash Z, Z \backslash X)=0$ by Lemma 5.5(c). This gives $i_{E \cup L}(X \cup Z \cup\{v\})=2|X \cup Z \cup\{v\}|+1$ so $X \cup Z=V-v$. The path $P$ now implies that $d(X \backslash Z, Z \backslash X)>0$, a contradiction. 
Hence we have $X \cap Z=\{r\}$ and, by symmetry, $X \cap Y=\{t\}$. This gives

$$
\begin{aligned}
i_{E \cup L}(X \cup Y \cup Z \cup\{v\}) & \geq i_{E \cup L}(X)+i_{E}(Y)+i_{E}(Z)+3 \\
& =2|X|+(2|Y|-3)+(2|Z|-3)+3 \\
& =2|X \cup Y \cup Z \cup\{v\}|+1 .
\end{aligned}
$$

Since $G$ is a rigid $\mathcal{M}_{l c}$-circuit, we must have $X \cup Y \cup Z \cup\{v\}=V$ and $i_{E \cup L}(X)+i_{E}(Y)+$ $i_{E}(Z)+3=|E|+|L|$. This implies that all loops in $G$ are contained in $G[X]$ and that the component of $G-\{r, t\}$ which contains $\{v, s\}$ has no loops. Hence $G$ is not balanced.

We call a triple $(X, Y, Z)$ of three sets satisfying the hypotheses of Lemma 5.7 for $G$ an unbalanced flower on $v$. Note that $(X, Y, Z)$ cannot be both an unbalanced flower and a strong or weak flower since, in the the former, $X \cap Y \cap Z=\emptyset$ and $G[Y], G[Z]$ are connected, while, for every strong or weak flower with $X \cap Y \cap Z=\emptyset$, each of $G[X], G[Y], G[Z]$ are disconnected.

Lemma 5.8. Let $G$ be a rigid $\mathcal{M}_{l c}$-circuit and $v$ be a non-admissible node of $G$ with three distinct neighbours. Then at least one of the following holds:

(a) there exists a strong flower on $v$ in $G$;

(b) there exsits a weak flower on $v$ in $G$;

(c) there exists an unbalanced flower on $v$ in $G$.

Proof. This follows immediately from Lemmas 5.4, 5.6 and 5.7.

Our final result of this section is a decomposition lemma for unbalanced rigid $\mathcal{M}_{l c}$-circuits i.e. rigid $\mathcal{M}_{l c}$-circuits which are not balanced. It uses the following graph operation.

Given three looped simple graphs $G=(V, E, L), G_{1}=\left(V_{1}, E_{1}, L_{1}\right)$ and $G_{2}=\left(V_{2}, E_{2}, L_{2}\right)$, we say that $G$ is the 2 -sum of $G_{1}$ and $G_{2}$ along an edge $u v$ if $V_{1} \cup V_{2}=V, V_{1} \cap V_{2}=\{u, v\}$, $E=\left(E_{1} \cup E_{2}\right)-u v, E_{1} \cap E_{2}=\{u v\}, L=L_{1} \cup L_{2}$ and $L_{1} \cap L_{2}=\emptyset$. Figure 6 gives an example of an unbalanced rigid $\mathcal{M}_{l c}$-circuit which is the 2-sum of a rigid and a flexible $\mathcal{M}_{l c}$-circuit. Lemma 5.9 below shows that every unbalanced rigid $\mathcal{M}_{l c}$-circuit can be obtained in this
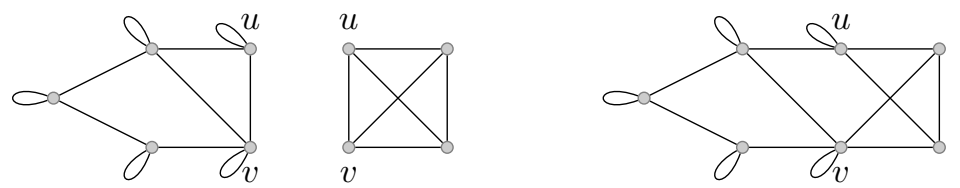

FigURE 6. An unbalanced rigid $\mathcal{M}_{l c}$-circuit on the right (removing $u$ and $v$ results in a loopless component) obtained from a rigid $\mathcal{M}_{l c}$-circuit and a flexible $\mathcal{M}_{l c}$-circuit by a 2 -sum operation along the edge $u v$ on the left.

way.

Lemma 5.9. Let $G=(V, E, L)$ be a looped simple graph. Then $G$ is an unbalanced rigid $\mathcal{M}_{l c}$-circuit if and only if $G$ is a 2 -sum of a rigid $\mathcal{M}_{l c}$-circuit and a flexible $\mathcal{M}_{l c}$-circuit.

Proof. First suppose that $G$ is a 2 -sum of a rigid $\mathcal{M}_{l c}$-circuit $G_{1}=\left(V_{1}, E_{1}, L_{1}\right)$ and a flexible $\mathcal{M}_{l c}$-circuit $G_{2}=\left(V_{2}, E_{2}\right)$ along an edge $e=u v \in E_{1} \cap E_{2}$. It is straightforward to check that $G$ satisfies the conditions given in Lemma 5.1(a). Hence $G$ is a rigid $\mathcal{M}_{l c}$-circuit. It is unbalanced since $G_{2}-\{u, v\}$ is a connected component of $G-\{u, v\}$ which contains no loops. 
Now suppose $G$ is an unbalanced, rigid $\mathcal{M}_{l c}$-circuit. Then there is a set $\{u, v\} \subset V$ such that $G-\{u, v\}$ has a component $H$ with no loops. Let $H_{1}=\left(V_{1}, E_{1}, L_{1}\right)$ be the subgraph of $G$ induced by $V \backslash V(H)$, and $H_{2}=\left(V_{2}, E_{2}\right)$ be the simple subgraph of $G$ obtained from $G-\left(V_{1} \backslash\{u, v\}\right)$ by deleting any loops at $u$ or $v$. We have

$$
2|V|+1=|E|+|L| \leq i_{E_{1} \cup L_{1}}\left(V_{1}\right)+i_{E_{2}}\left(V_{2}\right) \leq 2\left|V_{1}\right|+2\left|V_{2}\right|-3=2|V|+1
$$

Thus equality must occur throughout. This implies that $u v \notin E$ (by equality in the first inequality), $\left|E_{1}\right|+\left|L_{1}\right|=2\left|V_{1}\right|$ and $\left|E_{2}\right|=2\left|V_{2}\right|-3$ (by equality in the second inequality). Let $G_{1}$ and $G_{2}$ be the graphs obtained from $H_{1}$ and $H_{2}$, respectively, by adding the edge $u v$. It is straightforward to check that $G_{1}$ and $G_{2}$ satisify the conditions of Lemma 5.1 (a) and (b), respectively. Hence $G_{1}$ is a rigid $\mathcal{M}_{l c}$-circuit, $G_{2}$ is a flexible $\mathcal{M}_{l c}$-circuit, and $G$ is the 2 -sum of $G_{1}$ and $G_{2}$.

\section{6. $\mathcal{M}_{l c}$-CONNECTED GRAPHS}

Our long term aim is to obtain a recursive construction for balanced redundantly rigid graphs. To accomplish this we first consider the closely related family of ' $\mathcal{M}_{l c}$-connected graphs'. It is easy to see that a looped simple graph $G=(V, E, L)$ is redundantly rigid if and only if it is rigid and every element of $E \cup L$ belongs to an $\mathcal{M}_{l c}$-circuit. The graph $G$ is $\mathcal{M}_{l c}$-connected if every pair of elements of $E \cup L$ belong to a common $\mathcal{M}_{l c}$-circuit in $G$.

We will show that any balanced $\mathcal{M}_{l c}$-connected graph other than $K_{1}^{[3]}$ can be reduced to a smaller $\mathcal{M}_{l c}$-connected graph using the operation of edge/loop deletion or 1-reduction. Our proof uses the concept of an 'ear decomposition' of a matroid and follows a similar strategy to that used in [3, 11].

Recall that a matroid $M=(E, r)$ is connected if every pair of elements of $M$ is contained in a common circuit. Given a non-empty sequence of circuits $C_{1}, C_{2}, \ldots, C_{m}$ in $M$, let $D_{i}=C_{1} \cup C_{2} \cup \ldots C_{i}$ for all $1 \leq i \leq m$, and put $\tilde{C}_{i}=C_{i}-D_{i-1}$. The sequence $C_{1}, C_{2}, \ldots, C_{m}$ is a partial ear decomposition of $M$ if, for all $2 \leq i \leq m$,

(E1) $C_{i} \cap D_{i-1} \neq \emptyset$,

(E2) $C_{i}-D_{i-1} \neq \emptyset$, and

(E3) no circuit $C_{i}^{\prime}$ satisfying (E1) and (E2) has $C_{i}^{\prime}-D_{i-1} \subset C_{i}-D_{i-1}$.

A partial ear decomposition $C_{1}, C_{2}, \ldots, C_{m}$ is an ear decomposition of $M$ if $D_{m}=E$.

Lemma 6.1 ([7]). Let $M=(E, r)$ be a matroid with $|E| \geq 2$. Then:

(i) $M$ is connected if and only if $M$ has an ear decompostion.

(ii) If $M$ is connected then every partial ear decomposition is extendable to an ear decomposition of $M$.

(iii) If $C_{1}, C_{2}, \ldots, C_{m}$ is an ear decomposition of $M$ then $r\left(D_{i}\right)-r\left(D_{i-1}\right)=\left|\tilde{C}_{i}\right|-1$ for all $2 \leq i \leq m$.

Given a looped simple graph $G$, it will be convenient to refer to an ear decomposition $C_{1}, C_{2}, \ldots, C_{m}$ of $\mathcal{M}_{l c}(G)$ as an ear decomposition $H_{1}, H_{2}, \ldots, H_{m}$ of $G$ where $H_{i}$ is the $\mathcal{M}_{l c^{-}}$ circuit of $G$ induced by $C_{i}$ for $1 \leq i \leq m$. See Figure 7 for an example giving two distinct ear decompositions of the graph $G$ drawn on the far left. The ear decomposition drawn in the middle has a flexible $\mathcal{M}_{l c}$-circuit $K_{4}$ whereas the circuits of the ear decomposition drawn on the right are all rigid $\mathcal{M}_{l c}$-circuits. The following lemma tells that a rigid $\mathcal{M}_{l c}$-connected graph always has an ear decompositon into rigid $\mathcal{M}_{l c}$-circuits.

Lemma 6.2. Let $G$ be an $\mathcal{M}_{l c}$-connected looped simple graph with at least one loop. Then $G$ has an ear decomposition into rigid $\mathcal{M}_{l c}$-circuits. 

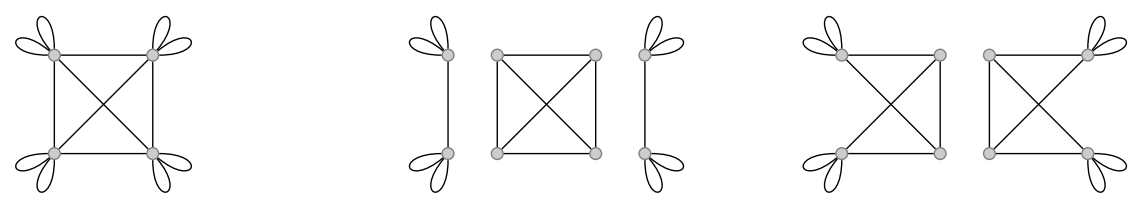

Figure 7. A graph $G$ on the left, and two ear decompositions of $G$ in the middle and on the right.

Proof. Let $\ell$ be a loop of $G$. Since $G$ is $\mathcal{M}_{l c}$-connected there exists an $\mathcal{M}_{l c}$-circuit $H_{1}$ containing $\ell$. Then $H_{1}$ is a rigid $\mathcal{M}_{l c}$-circuit and, if $G=H_{1}$, then we are done. So suppose $G \neq H_{1}$. Extend $H_{1}$ to an ear decomposition $H_{1}, H_{2}, \ldots, H_{k}$ of $G$ such that each $H_{i}$ is a rigid $\mathcal{M}_{l c}$-circuit for $1 \leq i \leq k$ and $k$ is as large as possible.

Suppose $k<m$. Then we may choose an edge or loop $f$ in $H_{k+1}$ which does not belong to $\bigcup_{i=1}^{k} H_{i}$. Since $\bigcup_{i=1}^{k+1} H_{i}$ is $\mathcal{M}_{l c}$-connected, there exists an $\mathcal{M}_{l c}$-circuit $H_{k+1}^{\prime} \subseteq \bigcup_{i=1}^{k+1} H_{i}$ such that $\ell, f$ are in $H_{k+1}^{\prime}$. Then $H_{k+1}^{\prime}$ is a rigid $\mathcal{M}_{l c}$-circuit and $H_{1}, H_{2}, \ldots, H_{k}, H_{k+1}^{\prime}$ is a partial ear decomposition of $G$. Since every partial ear decomposition can be extended to a 'full' ear decomposition, this contradicts the maximality of $k$.

Lemma 6.2 and the fact that the union of two redundantly rigid graphs is redundantly rigid immediately give

Corollary 6.3. Let $G$ be an $\mathcal{M}_{l c}$-connected looped simple graph. Then $G$ is redundantly rigid if and only if $G$ has a loop.

Lemma 6.4. Let $G$ be an $\mathcal{M}_{l c}$-connected looped simple graph that contains a loop. Let $C_{1}, C_{2}, \ldots, C_{m}$ be an ear decomposition of $\mathcal{M}_{l c}(G)$ where $m \geq 2$ and $H_{i}=G\left[C_{i}\right]$ is a rigid $\mathcal{M}_{l c}$-circuit for $1 \leq i \leq m$. Let $Y=V\left(H_{m}\right) \backslash \bigcup_{i=1}^{m-1} V\left(H_{i}\right)$ and $X=V\left(H_{m}\right) \backslash Y$. Then

(i) $\left|\tilde{C}_{m}\right|=2|Y|+1$;

(ii) if $Y \neq \emptyset$ then every edge/loop e $\in \tilde{C}_{m}$ is incident to $Y, X$ is mixed critical in $H_{m}$ and $G[Y]$ is connected;

(iii) if $G$ is balanced, $Y \neq \emptyset$ and $\tilde{C}_{m}$ contains no loops then $Y$ has at least three neighbours in $X$.

Proof. (i) Let $G_{j}=\bigcup_{i=1}^{j} H_{i}$ and $D_{j}=\bigcup_{i=1}^{j} C_{j}$. Hence $E\left(G_{j}\right) \cup L\left(G_{j}\right)=D_{j}$. Lemma 6.1(i) implies that $G_{m-1}$ is $\mathcal{M}_{l c}$-connected. Corollary 6.3 implies that $G_{m-1}$ and $G$ are rigid so $r\left(D_{m-1}\right)=2|V \backslash Y|$ and $r(E \cup L)=2|V|$. Hence by Lemma 6.1(iii) we have $\left|\tilde{C}_{m}\right|=r(E \cup L)-r\left(D_{m-1}\right)+1=2|V|-2|V-Y|+1=2|Y|+1$.

(ii) Suppose $Y \neq \emptyset$. Let $k$ be the number of edges/loops in $E \cup L-D_{m-1}$ which have all endvertices in $V\left(G_{m-1}\right)$. Since $H_{m}$ is a rigid $\mathcal{M}_{l c}$-circuit, part (i) implies that

$$
i_{H_{m}}(X)=\left|C_{m}\right|-\left|\tilde{C}_{m}\right|+k=2|X \cup Y|+1-(2|Y|+1)+k=2|X|+k .
$$

Since $H_{m}[X]$ is a proper subgraph of $H_{m}$ we must have $k=0$ and $X$ is mixed critical in $H_{m}$.

Assume $G[Y]$ is disconnected. Let $Y_{1}, Y_{2}, \ldots, Y_{k}$ be the vertex sets of the connected components of $G[Y]$. Since $H_{m}$ is an $\mathcal{M}_{l c}$-circuit, $k \geq 2$ and $X$ is mixed critical, we have $i_{H_{m}}\left(X \cup Y_{i}\right)-i_{H_{m}}(X) \leq 2\left|X \cup Y_{i}\right|-2|X|=2\left|Y_{i}\right|$. This implies that

$$
\left|\tilde{C}_{m}\right|=\sum_{i=1}^{k}\left(i_{H_{m}}\left(X \cup Y_{i}\right)-i_{H_{m}}(X)\right) \leq \sum_{i=1}^{k} 2\left|Y_{i}\right|=2|Y|,
$$


contradicting part (i).

(iii) Let $X^{\prime}$ be the set of vertices in $X$ which are adjacent to $Y$. Then $G-X^{\prime}$ has a component with no loops. The fact that $G$ is balanced and $\mathcal{M}_{l c}$-connected now implies that $\left|X^{\prime}\right| \geq 3$.

Lemma 6.5. Let $G=(V, E, L)$ be an $\mathcal{M}_{l c}$-connected looped simple graph which contains a loop. Suppose that $G^{\prime}$ is obtained from $G$ by an edge or loop addition, or a 1-extension. Then $G^{\prime}$ is $\mathcal{M}_{l c}$-connected.

Proof. Note that $G$ is redundantly rigid by Corollary 6.3 .

First suppose that $G^{\prime}$ is obtained from $G$ by adding a new edge or loop $f$. Since $G$ is rigid, there exists an $\mathcal{M}_{l c}$-circuit $C$ in $G^{\prime}$ with $f \in C$. The $\mathcal{M}_{l c}$-connectivity of $G^{\prime}$ now follows from the transitivity of the relation that defines $\mathcal{M}_{l c}$-connectivity.

Next suppose $G^{\prime}$ is obtained from $G$ by a 1-extension operation that deletes an edge or loop $f$ and adds a new vertex $v$ with three incident edges or loops $f_{1}, f_{2}, f_{3}$. By transitivity, it will suffice to show that every $e \in(E \cup L)-f$ belongs to an $\mathcal{M}_{l c^{-}}$-circuit of $G^{\prime}$ containing $f_{1}, f_{2}$ and $f_{3}$. Since $G$ is $\mathcal{M}_{l c}$-connected, there exists an $\mathcal{M}_{l c}$-circuit $C$ in $G$ containing $e$ and $f$. Choose a base $B$ of $\mathcal{M}_{l c}(G-f)$ with $C-f$ contained in $B$. Then $G[B]$ is rigid simce $G$ is rigid and $e \in C \subseteq B+f$. Hence $B^{\prime}=B-e+f$ is another base of $\mathcal{M}_{l c}(G)$ and $G\left[B^{\prime}\right]$ is rigid. Then $B^{\prime \prime}=B^{\prime}-f+f_{1}+f_{2}+f_{3}$ is a base of $\mathcal{M}_{l c}\left(G^{\prime}\right)$ and $G^{\prime}\left[B^{\prime \prime}\right]$ is rigid, since $G^{\prime}\left[B^{\prime \prime}\right]$ is obtained by performing a 1-extension on $G\left[B^{\prime}\right]$. Hence $B^{\prime \prime}+e$ contains a unique $\mathcal{M}_{l c}$-circuit $C^{\prime}$. Since $B^{\prime \prime}$ is independent we have $e \in C^{\prime}$. If $f_{1} \notin C^{\prime}$ then, since $v$ is incident with exactly three edges, we would have $C \subseteq B$, contradicting the fact that $B$ is independent. Hence $f_{1}$ is in $C^{\prime}$ and, since $v$ is incident to exactly three edges, we also have $f_{2}, f_{3} \in C^{\prime}$.

We next consider the inverse operations to edge/loop addition and 1-extension. To do this we extend our definition of admissibility from an $\mathcal{M}_{l c}$-circuit to an $\mathcal{M}_{l c}$-connected graph $G=(V, E, L)$. We say that an edge or loop $f \in E \cup L$ is admissible if $G-f$ is $\mathcal{M}_{l c^{-c o n n e c t e d}}$, and a node $v \in V$ is admissible if there exists a 1-reduction at $v$ which results in an $\mathcal{M}_{l c^{-}}$ connected graph.

We will show that every balanced $\mathcal{M}_{l c^{-}}$connected graph $G$ other than $K_{1}^{[3]}$ has an admissible edge or node. The main idea is to find an admissible edge/loop or node in the last $\mathcal{M}_{l c}$-circuit of an ear decomposition of $G$. We will need some additional terminology for nodes in rigid $\mathcal{M}_{l c}$-circuits to do this.

Suppose $v$ is a node in a rigid $\mathcal{M}_{l c}$-circuit $G=(V, E, L)$ and $X$ is a critical set in $G$. We say that $X$ is node critical for $v$ if $N(v)-z \subseteq X \subseteq V \backslash\{v, z\}$ for some $z \in N(v)$ with $d^{\dagger}(z) \geq 4$. We will also refer to a critical set $X$ as being node critical when it is node critical for some (unspecified) node. We say that $v$ is a leaf node if $d_{G^{\dagger}\left[V_{3}\right]}(v) \in\{0,1\}$, a series node if $d_{G^{\dagger}\left[V_{3}\right]}(v)=2$ and a branching node if $d_{G^{\dagger}\left[V_{3}\right]}(v)=3$. Note that a 1-reduction at a branching node cannot be admissible since the resulting graph will have a vertex $v$ with $d^{\dagger}(v)=2$.

Theorem 6.6. Let $G=(V, E, L)$ be a balanced, $\mathcal{M}_{l c}$-connected, looped simple graph distinct from $K_{1}^{[3]}$. Then $G$ has an admissible edge or loop, or an admissible node.

Proof. We proceed by contradiction. Suppose that $G$ has no admissible edges or nodes. Since $G$ is balanced, $G$ has at least one loop. Lemma 6.2 now implies that $G$ has an ear decomposition $C_{1}, C_{2}, \ldots, C_{m}$ such that $H_{i}=G\left[C_{i}\right]$ is a rigid $\mathcal{M}_{l c}$-circuit for all $1 \leq i \leq m$. Let $H_{i}=\left(V_{i}, E_{i}, L_{i}\right)$ for all $1 \leq i \leq m$ and $H=\bigcup_{i=1}^{m-1} H_{i}$. Then $H$ is $\mathcal{M}_{l c}$-connected by Lemma 6.1(i). Put $Y=V_{m} \backslash \cup_{i=1}^{m-1} V_{i}$ and $X=V_{m} \backslash Y$. Note that if $m=1$ then $Y=V$ 
and $X=\emptyset$. If $Y=\emptyset$ then $H=G-e$ for some $e \in E \cup L$ and $e$ is admissible in $G$ since $H$ is $\mathcal{M}_{l c}$-connected. Hence we may assume that $Y \neq \emptyset$. Lemma 6.4(ii) now implies that $X$ is mixed critical in $H_{m}$. Hence $Y$ contains at least one node of $H_{m}$ by Lemma 5.3

Claim 6.7. No node of $H_{m}$ in $Y$ is admissible in $H_{m}$, and hence every node of $H_{m}$ in $Y$ has at least two distinct neighbours in $G$.

Proof. Suppose some node $v \in Y$ is admissible in $H_{m}$. Let $C_{m}^{\prime}$ be the edge set of the rigid $\mathcal{M}_{l c}$-circuit obtained from $H_{m}$ by performing an admissible 1-reduction at $v$. Then $v$ is a node in $G$ and the graph obtained from $G$ by performing the same 1-reduction at $v$ will be $\mathcal{M}_{l c}$-connected since it will have an ear decomposition $C_{1}, C_{2}, \ldots, C_{m-1}, C_{m}^{\prime}$. Hence $v$ will be admissible in $G$, contradicting the fact that $G$ has no admissible nodes. We can now use lemma 5.4 (c) to deduce that $v$ has at least two distinct neighbours.

Claim 6.8. Suppose $v$ is a node of $H_{m}$ in $Y$. Then $v$ has at least two neighbours in $Y$.

Proof. We split the proof into three cases.

Case 1: $N(v) \cap Y=\emptyset$.

Since $H_{m}[Y]$ is connected by Lemma 6.4(ii), we have $Y=\{v\}$. If $G[N(v)]$ is not complete then we may choose $p, q \in N(v)$ with $p q \notin E$. Then the 1-reduction at $v$ which adds the edge $p q$ creates the graph $H+p q$. Lemma 6.5 and the fact that $H$ is $\mathcal{M}_{l c}$-connected now imply that this 1-reduction is admissible in $G$. This contradicts the assumption that $G$ has no admissible nodes. Hence $G[N(v)]$ is complete. Then $G-v=H$ by Lemma 6.4(ii), so $G-v$ is $\mathcal{M}_{l c}$-connected. Since $G-e$ is a 1-extension of $H$ for any edge $e$ in $G[N(v)]$, it is also $\mathcal{M}_{l c}$-connected by Lemma 6.5. Hence $e$ is admissible in $G$. This contradicts the assumption that $G$ has no admissible edges.

Case 2: $N(v)=\{r, s, t\}$ with $\{r, t\} \subseteq X, s \in Y$.

Since $v$ is not admissible in $H_{m}$, there exist critical sets $R, T$ in $H_{m}$ such that $\{s, r\} \subseteq$ $T \subseteq V_{m} \backslash\{v, t\}$ and $\{s, t\} \subseteq R \subseteq V_{m} \backslash\{v, r\}$ by Lemma [5.4. We may suppose that $R, T$ have been chosen to be the minimal critical sets with these properties. If $R, T$ are both pure critical, then $(R, S, X)$ would be an unbalanced flower on $v$ in $H_{m}$, and we could use Lemma 5.7 to contradict the hypothesis that $G$ is balanced. Relabelling $R, T$ if necessary we may assume that $R$ is mixed critical, but not pure critical, in $H_{m}$. Lemma 5.8 now implies that $(X, R, T)$ is a strong or weak flower on $v$ in $H_{m}$. Let $H_{m}^{\prime}$ denote the graph obtained from $H_{m}$ by applying a 1-reduction at $v$ adding the edge $s t$. Then $H_{m}^{\prime}$ contains a unique $\mathcal{M}_{l c}$-circuit $D$. The minimality of $R$ and the fact that $R$ is not pure critical now imply that $D=H_{m}[R]+s t$ and $D$ is a rigid $\mathcal{M}_{l c}$-circuit. Let $C_{m}^{\prime}=E(D) \cup L(D)$.

We will show that $v$ is admissible in $G$ by verifying that $G^{\prime}=G-v+s t$ is $\mathcal{M}_{l c}$-connected. Since $H$ is $\mathcal{M}_{l c}$-connected, it will suffice to show that $G^{\prime}=H \cup D$ and that $D$ contains an edge or loop of $H$. We first verifiy that $D$ contains an edge of $H$. Since each edge of $\tilde{C}_{m}$ is incident with $Y$ by Lemma 6.4(ii), this is equivalent to showing that $D$ contains an edge of $H_{m}[X]$. This follows because $R$ and $X$ are mixed critical in $H_{m}$, so $R \cap X$ is mixed critical and nonempty, and all edges of $H_{m}[R \cap X]$ belong to $D$. It remains to show that $G^{\prime}=D \cup H$ i.e. $\left(\tilde{C}_{m} \backslash\{v r, v s, v t\}\right)+s t \subseteq C_{m}^{\prime}$. Lemma 5.6 implies that all edges of $H_{m}$ which are not incident with $v$ are induced by either $X$ or $R$, and no edge of $\tilde{C}_{m}$ is induced by $X$ by Lemma 6.4(ii). Since $C_{m}^{\prime}=E(D) \cup L(D)$ and $D=H_{m}[R]+s t$ we have $\left(\tilde{C}_{m} \backslash\{v r, v s, v t\}\right)+s t \subseteq C_{m}^{\prime}$. Thus $G^{\prime}$ is $\mathcal{M}_{l c}$-connected and $v$ is admissible in $G$.

Case 3: $N(v)=\{r, s\}$ with $r \in X$ and $s \in Y$.

Since $v$ is not admissible in $H_{m}$, Lemma 5.4 implies that there exists a pure critical set $R$ in $H_{m}$ with $\{r, s\} \subseteq R \subseteq V\left(H_{m}\right)-v$ and a mixed critical set $S$ with $s \in S \subseteq V\left(H_{m}\right) \backslash\{r, v\}$. We may assume that $R$ and $S$ have been chosen to be the minimal such sets. 
Suppose that $|R \cap X| \geq 2$. Then $X \cup R$ is mixed critical, $X \cap R$ is pure critical, $H_{m}[R]$ is simple and $H_{m}-v=H_{m}[X] \cup H_{m}[R]$ by Lemma 5.5(c). Note that Lemma 5.5(b) applied to $X$ and $S$ tells us that $r s \notin E$. Let $H_{m}^{\prime}=H_{m}-v+r s$. Then $H_{m}^{\prime}$ contains a unique $\mathcal{M}_{l c}$-circuit $D$. The minimality of $R$ now implies that $D=H_{m}[R]+r s$ and $D$ is simple. Let $G^{\prime}=G-v+r s$. We will show that $G^{\prime}$ is $\mathcal{M}_{l c^{-}}$-connected. Since $H_{m}-v=H_{m}[X] \cup H_{m}[R]$ and $X \subseteq V(H)$, all edges of $G^{\prime}$ belong to $H$ or $D$. Since $H$ and $D$ are both $\mathcal{M}_{l c}$-connected and have at least one edge in common (as $X \cap R$ is a critical set with at least two vertices in $\left.H_{m}\right), G^{\prime}$ is $\mathcal{M}_{l c}$-connected and hence $v$ is admissible in $G$. This contradicts the assumption that $G$ has no admissible nodes so we must have $R \cap X=\{r\}$.

Since $R$ is pure-critical, $R \cap X=\{r\}$ and $s \in R$, we can find a path $P$ in $H_{m}[R]$ from $r$ to $s$ which avoids $X-r$. Since $X$ and $S$ are mixed critical in $H_{m}$ and $v \notin X \cup S$, Lemma 5.5(b) implies there are no edges in $H_{m}$ from $X \backslash S$ to $S \backslash X$. Since $r \in X \backslash S$ and $s \in S \backslash X$, this contradicts the existence of the path $P$.

Claim 6.9. Let $v$ be a node of $H_{m}$ in $Y$ with three distinct neighbours. Then there is no unbalanced flower on $v$ in $H_{m}$.

Proof. Let $N(v)=\{w, u, z\}$ and suppose there exists an unbalanced flower $(W, U, Z)$ on $v$ in $H_{m}$ with $u, z \in W \subseteq V_{m} \backslash\{v, w\}, w, z \in U \subseteq V_{m} \backslash\{v, u\}$, and $w, u \in Z \subseteq V_{m} \backslash\{v, z\}$.

First suppose that $N(v) \subseteq Y$. We may assume that $U, Z$ are pure critical and that $W$ is mixed critical. Replacing $W$ by $W \cup X$ if necessary, we may assume that $X \subseteq W$. The properties of an unbalanced flower (Lemma 5.7) now tell us that the component of $G-\{u, z\}$ which contains $\{v, w\}$ is loopless. This contradicts the hypothesis that $G$ is balanced.

Now suppose $N(v) \nsubseteq Y$. Claim 6.8 implies that $v$ has at least two neighbours in $Y$. Hence we may assume that $u, z \in Y$ and $w \in X$. Since $X$ is mixed critical, each connected component of $H_{m}[X]$ contains a loop. Lemma 5.7 and the fact that $X \cap Z=\{w\} \subseteq X \cap U$, now implies that $U$ is mixed critical, $W$ is pure critical, and no vertex of $(W \cup Z) \backslash\{w, z\}$ is incident with a loop in $H_{m}$. The facts that $H_{m}[X]$ is rigid and no vertex of $X \cap(W-z)$ is incident with a loop now imply that $X \cap(W-z)=\emptyset$, and hence $X \subseteq U$. This contradicts the hypothesis that $G$ is balanced since the component of $G-\{w, z\}$ which contains $\{v, u\}$ will be loopless.

Claim 6.10. We can choose a node $v$ of $H_{m}$ in $Y$ and a critical set $X_{v}$ in $H_{m}$ such that $X_{v}$ is mixed node critical for $v$ and $X \subseteq X_{v}$.

Proof. Let $F=H_{m}^{\dagger}\left[V_{3} \cap Y\right]$. Then $F$ is a forest by Lemma 5.2 and we may choose a vertex $v$ of $F$ such that $d_{F}(v) \leq 1$. Since $v$ has at least two neighbours in $Y$ by Claim 6.8, $v$ has a neighbour $z$ in $Y$ with $d^{\dagger}(z) \geq 4$.

We first consider the case when $N(v)=\{y, z\}$. Then $y \in Y$ by Claim 6.8. Since $v$ is not admissible in $H_{m}$, Lemma 5.4 implies that there exists a mixed critical set $S$ in $H_{m}$ with $y \in S \subseteq V_{m} \backslash\{v, z\}$. Then $S \cup X$ is a mixed node critical set for $v$.

We next consider the case when $N(v)=\{w, u, z\} \subseteq Y$. Since $d_{F}(v) \leq 1$ we may assume that $d^{\dagger}(u) \geq 4$. Since $v$ is not admissible in $H_{m}$, Lemma 5.8 and Claim 6.9 imply that there exists a strong or weak flower $(W, U, Z)$ on $v$ in $H_{m}$ with $u, z \in W \subseteq V_{m} \backslash\{v, w\}$, $w, z \in U \subseteq V_{m} \backslash\{v, u\}$, and $w, u \in Z \subseteq V_{m} \backslash\{v, z\}$. Then either $Z$, or $U$, is mixed critical and hence either $Z \cup X$, or $U \cup X$, is a mixed node critical set for $v$.

It remains to consider the case when $N(v)=\{w, u, z\} \nsubseteq Y$. Since $v$ has at least two neighbours in $Y$ we may assume that $u \in Y$ and $w \in X$. Since $v$ is not admissible in $H_{m}$, Lemma 5.8 and Claim 6.9 imply that there exists a strong or weak flower $(W, U, Z)$ on $v$ in $H_{m}$ with $u, z \in W \subseteq V_{m} \backslash\{v, w\}, w, z \in U \subseteq V_{m} \backslash\{v, u\}$, and $w, u \in Z \subseteq V_{m} \backslash\{v, z\}$. If 
$Z$ is mixed critical or $|X \cap Z| \geq 2$ then $Z \cup X$ is a mixed node critical set for $v$. Hence we may assume that $Z$ is not mixed critical and $X \cap Z=\{w\}$.

Thus $(W, U, Z)$ is a weak flower. Lemma 5.6 and the fact that $Z$ is not mixed critical now imply that $W$ is mixed critical and $i_{L}\left(W^{\dagger}\right)=0=d\left(W^{\dagger}, Z^{\dagger}\right)$. Furthermore, since $X \cap Z=\{w\}$, we have $X-w \subseteq Z^{\dagger}$. This implies that $w$ is an isolated vertex of $H_{m}[X]$ and contradicts the fact that $X$ is mixed critical in $H_{m}$.

Choose a node $v$ of $H_{m}$ in $Y$ and a mixed node critical set $X_{v}$ for $v$ in $H_{m}$ which satisfy the conditions of Claim 6.10 and are such that $\left|X_{v}\right|$ is as large as possible over all such choices of $v$ and $X_{v}$. Let $Y_{v}=V_{m} \backslash X_{v}$. Since $X_{v}$ is mixed node critical for $v,\left|Y_{v}\right| \geq 2$. Let $F_{v}=H_{m}^{\dagger}\left[V_{3} \cap Y_{v}\right]$. By Lemma 5.3 we can choose a node $z \neq v$ in $Y_{v}$ such that $d_{F_{v}}(z) \leq 1$. By Claim 6.7, $z$ has at least two distinct neighbours in $G$.

Suppose $z$ has exactly two neighbours, say $a, b$, in $G$. If $\{a, b\} \cap X_{v} \neq \emptyset$ then the set $X_{v}^{\prime}=X_{v}+z$ would contradict the maximality of $X_{v}$. Hence $\{a, b\} \subset Y_{v}$ and since $d_{F_{v}}(z) \leq 1$ we may assume that $d^{\dagger}(b) \geq 4$. Since $z$ is not admissible in $H_{m}$ we have $a \in X_{z} \subseteq V \backslash\{z, b\}$ for some mixed critical set $X_{z}$. Then $X_{z}^{\prime}=X_{z} \cup X_{v}$ is mixed node critical for $z$ and contradicts the maximality of $X_{v}$. Hence $z$ has three distinct neighbours in $G$.

The facts that $H_{m}$ is an $\mathcal{M}_{l c}$-circuit, $X_{v}$ is mixed critical and $v \notin X_{v}+z$ imply that $z$ has at most two neighbours in $X_{v}$. If $z$ had exactly two neighbours in $X_{v}$, then $X_{v} \cup\{z\}$ would be a mixed node critical set for $v$ and would contradict the maximality of $X_{v}$. Hence $z$ has at most one neighbour in $X_{v}$. Consider the following two cases.

Case 1: $z$ is a series node in $H_{m}$.

Let $N(z)=\{p, q, t\}$. Since $z$ is a series node in $H_{m}$ and $d_{F_{v}}(z) \leq 1, z$ has exactly one neighbour, say $t$, in $X_{v}$ and $t$ is a node in $H_{m}$. Without loss of generality, we may assume $d^{\dagger}(p)=3$ and $d^{\dagger}(q) \geq 4$. Since $z$ is non-admissible, Lemma 5.4(a) implies there exists a (pure or mixed) critical set $X_{z}$ with $\{t, p\} \subseteq X_{z} \subseteq V \backslash\{z, q\}$. Since $H_{m}^{\dagger}\left[V_{3}\right]$ is a forest by Lemma 5.2, we have $p t \notin E$ and hence $X_{z} \neq\{t, p\}$. Since $X_{v}, X_{z}$ are critical, $t$ is a node, $t \in X_{v} \cap X_{z}$ and $z \notin X_{v} \cup X_{z}$, all neighbours of $t$ other than $z$ belong to $X_{v} \cap X_{z}$. Lemma 5.5(b) or (c) now implies that $X_{v} \cup X_{z}$ is mixed critical. Since $\{t, p\} \subseteq X_{v} \cup X_{z} \subseteq V \backslash\{z, q\}$ and $d^{\dagger}(q) \geq 4, X_{v} \cup X_{z}$ is mixed node critical for $z$. This contradicts the maximality of $X_{v}$.

Case 2: $z$ is a leaf node in $H_{m}$.

Let $N(z)=\left\{z_{1}, z_{2}, z_{3}\right\}$. Since $z$ is non-admissible, Lemma 5.8 and Claim 6.9 imply there is either a strong or weak flower $\left(Z_{1}, Z_{2}, Z_{3}\right)$ on $z$ in $H_{m}$ with $Z_{i} \subseteq V_{m} \backslash\left\{z, z_{i}\right\}$ for $1 \leq i \leq 3$. We have $z_{i} z_{j} \notin E$ for all $1 \leq i<j \leq 3$ by Lemma 5.6(b) (since $z_{i} \in Z_{i}^{\dagger}$ ). Since $z$ is a leaf node in $H_{m}$, we may suppose that $d^{\dagger}\left(z_{1}\right) \geq 4$ and $d^{\dagger}\left(z_{2}\right) \geq 4$. Since neither $z_{1}$ nor $z_{2}$ are nodes in $H_{m}, Z_{1}$ and $Z_{2}$ are two node critical sets for $z$ with $Z_{1} \cup Z_{2}=V_{m}-z$ (by Lemma 5.6(a)) and at least one of them, say $Z_{1}$, is mixed critical. Then $Z_{1} \cup X_{v}$ is mixed critical. If $z_{1} \notin X_{v}$ then $Z_{1} \cup X_{v}$ would be a mixed node critical set for $z$ which would be larger than $X_{v}$ since $z_{2}, z_{3} \in Z_{1}$ and $\mid N(z) \cap X_{v} \| \leq 1$. This would contradict the maximality of $X_{v}$ so we must have $z_{1} \in X_{v}$. The fact that $\left|N(z) \cap X_{v}\right| \leq 1$ now implies that $z_{2}, z_{3} \notin X_{v}$. It follows that, if either $Z_{2}$ is mixed critical or $\left|Z_{2} \cap X_{v}\right| \geq 2$, then $Z_{2} \cup X_{v}$ would be a mixed node critical set for $z$ which is larger than $X_{v}$. Hence $Z_{2}$ is pure critical and $Z_{2} \cap X_{v}=\left\{z_{1}\right\}$.

We complete the proof by using a similar argument to the last paragraph of the proof of Claim 6.10. Lemma 5.6 and the fact that $Z_{2}$ is not mixed critical imply that $\left(Z_{1}, Z_{2}, Z_{3}\right)$ is a weak flower, and $i_{L}\left(Z_{2}^{\dagger}\right)=0=d\left(Z_{1}^{\dagger}, Z_{2}^{\dagger}\right)$. Furthermore, since $X_{v} \cap Z_{2}=\left\{z_{1}\right\}$, we have $X_{v}-z_{1} \subseteq Z_{2}^{\dagger}$. This implies that $z_{1}$ is an isolated vertex of $H_{m}\left[X_{v}\right]$ and contradicts the fact that $X_{v}$ is mixed critical in $H_{m}$. 
Recursive constructions. We close this section by using Theorem 6.6 to obtain a recursive construction for rigid $\mathcal{M}_{l c}$-connected graphs. It uses the special case of the 2-sum operation in which one side of the 2 -sum is a copy of $K_{4}$. We will refer to this operation and its inverse as a $K_{4}$-extension and a $K_{4}$-reduction, respectively.

We will need the following result on admissible reductions of loopless $\mathcal{M}_{l c}$-connected graphs and an extension of Lemma 5.9 to $\mathcal{M}_{l c}$-connected graphs.

Theorem 6.11. Let $G=(V, E)$ be an $\mathcal{M}_{l c}$-connected simple graph which is distinct from $K_{4}$ and $u v, x y \in E$.

(a) If $G$ is an $\mathcal{M}_{l c}$-circuit then some vertex of $V \backslash\{u, v, x\}$ is an admissible node in $G$.

(b) If $G$ is not an $\mathcal{M}_{l c}$-circuit then either some edge of $E \backslash\{u v, x y\}$ is admissible in $G$, or some vertex of $V \backslash\{u, v, x, y\}$ is an admissible node in $G$.

Proof. Part (a) follows immediately from [1, Theorem 3.8]. To see (b) we choose an $\mathcal{M}_{l c^{-}}$ circuit $H_{1}$ in $G$ containing $u v, x y$ and then extend $H_{1}$ to an ear decomposition $H_{1}, H_{2}, \ldots, H_{m}$ of $G$. Then [11, Theorem 5.4] implies that either some edge of $H_{m}$ distinct from $u v, x y$ is admissible in $G$ or some vertex of $H_{m}-\bigcup_{i=1}^{m-1} H_{m-1}$ is an admissible node of $G$.

An unbalanced 2-separator of a looped simple graph $G$ is a pair of vertices $\{u, v\}$ such that $G-\{u, v\}$ has a component with no loops. Note that we allow this loopless component to be equal to $G-\{u, v\}$. An unbalanced 2-separation of $G$ is an ordered pair of subgraphs $\left(G_{1}, G_{2}\right)$ such that $G=G_{1} \cup G_{2},\left|V\left(G_{1}\right) \cap V\left(G_{2}\right)\right|=2<\left|V\left(G_{2}\right)\right|$ and $E\left(G_{1}\right) \cap E\left(G_{2}\right)=\emptyset=L\left(G_{2}\right)$.

Lemma 6.12. Let $G=(V, E, L)$ be a looped simple graph with $L \neq \emptyset$, and $\left(G_{1}, G_{2}\right)$ be an unbalanced 2-separator of $G$ such that $V\left(G_{1}\right) \cap V\left(G_{2}\right)=\{u, v\}$ and $u v \notin E$. Then the following statements are equivalent:

(a) $G+u v$ is $\mathcal{M}_{l c}$-connected;

(b) $G_{1}+u v$ and $G_{2}+u v$ are both $\mathcal{M}_{l c}$-connected;

(c) $G$ is $\mathcal{M}_{l c^{-}}$connected.

Proof. Let $G_{i}+u v=\left(V_{i}, E_{i}, L_{i}\right)$ for $i=1,2$. By symmetry we may suppose $L_{1} \neq \emptyset=L_{2}$. Choose $f_{1} \in L_{1}$ and $g_{2} \in E_{2}-u v$.

(a) $\Rightarrow$ (b). Suppose that $G+u v$ is $\mathcal{M}_{l c}$-connected. Then there exists an $\mathcal{M}_{l c}$-circuit $C$ in $G+u v$ containing $f_{1}, g_{2}$. Lemma 5.9 now implies that $C$ is the 2-sum of two $\mathcal{M}_{l c}$-circuits $C_{1}, C_{2}$ with $f_{1}, u v \in C_{1} \subseteq G_{1}+u v$ and $g_{2}, u v \in C_{2} \subseteq G_{2}+u v$. Transitivity now implies that $G_{2}+u v$ is $\mathcal{M}_{l c}$-connected and that all loops of $G_{1}+u v$ belong the the same $\mathcal{M}_{l c}$-connected component $H_{1}$ of $G_{1}+u v$ as $u v$. To complete the proof we choose an edge $g_{1} \in E_{1}$ and show $g_{1}$ is in $H_{1}$. Since $G+u v$ is $\mathcal{M}_{l c}$-connected, there exists an $\mathcal{M}_{l c}$-circuit $C^{\prime}$ in $G+u v$ containing $f_{1}, g_{1}$. If $C^{\prime} \subseteq G_{1}+u v$ then we are done. On the other hand, if $C^{\prime} \nsubseteq G_{1}+u v$ then Lemma 5.9 implies that $\left(C^{\prime} \cap G_{1}\right)+u v$ is an $\mathcal{M}_{l c}$-circuit in $G_{1}+u v$ containing $f_{1}, g_{1}, u v$. (b) $\Rightarrow(\mathrm{c})$. Suppose $G_{1}+u v$ and $G_{2}+u v$ are both $\mathcal{M}_{l c}$-connected. Then there exists an $\mathcal{M}_{l c}$-circuit $C_{i}$ in $G_{i}+u v$ such that $C_{1}$ contains $f_{1}, u v$ and $C_{2}$ contains $g_{2}, u v$. By Lemma 5.9. $C=\left(C_{1}-u v\right) \cup\left(C_{2}-u v\right)$ is an $\mathcal{M}_{l c}$-circuit in $G$. Transitivity now implies that all loops of $G_{1}$ and all edges of $G_{2}$ belong the the same $\mathcal{M}_{l c}$-connected component $H$ of $G$. To complete the proof we choose an edge $g_{1} \in E_{1}-u v$ and show $g_{1}$ is in $H_{1}$. Since $G_{1}+u v$ is $\mathcal{M}_{l c}$-connected, there exists an $\mathcal{M}_{l c}$-circuit $C_{1}^{\prime}$ in $G+u v$ containing $f_{1}, g_{1}$. If $C_{1}^{\prime} \subseteq G$ then we are done. On the other hand, if $C_{1}^{\prime} \nsubseteq G_{1}+u v$ then $u v \in C_{1}^{\prime}$ and Lemma 5.9 implies that $\left(C_{1}^{\prime}-u v\right) \cup\left(C_{2}-u v\right)$ is an $\mathcal{M}_{l c}$-circuit in $G$ containing $f_{1}, g_{1}$.

(c) $\Rightarrow$ (a). This follows immediately from Lemma 6.5,

We can now give our recursive construction. 
Theorem 6.13. A looped simple graph is rigid and $\mathcal{M}_{l c}$-connected if and only if it can be obtained from $K_{1}^{[3]}$ by recursively applying the operations of 1 -extension, $K_{4}$-extension and adding a new edge or loop.

Proof. Sufficiency follows from Lemma 6.5 and the fact that $K_{1}^{[3]}$ is $\mathcal{M}_{l c^{-c o n n e c t e d . ~ T o ~}}$ prove necessity it will suffice to show that every rigid $\mathcal{M}_{l c}$-connected graph $G$ with at least two vertices can be reduced to a smaller rigid $\mathcal{M}_{l c}$-connected graph by applying either a 1-reduction, a $K_{4}$-reduction or an edge deletion. If $G$ is balanced then Theorem6.6 implies there is an edge, loop or node such that the corresponding edge/loop deletion or 1-reduction gives a rigid $\mathcal{M}_{l c}$-connected graph.

Hence we may assume that $G$ is unbalanced. By Lemma 6.12, $G$ is the 2-sum of an $\mathcal{M}_{l c}$-connected graph $G_{1}$ with at least one loop and a $\mathcal{M}_{l c}$-connected graph $G_{2}$ with no loops along an edge $u v$. We may assume that $G_{2}$ is 3 -connected by choosing a 2-sum such that $G_{2}$ is as small as possible. If $G_{2}=K_{4}$, then $G$ can be reduced to $G_{1}$ by applying the $K_{4}$-reduction operation.

Hence we may assume that $G_{2} \neq K_{4}$. Then Theorem6.11 implies that $G_{2}$ contains either an admissible edge distinct from $u v$, or an admissible node $x$ distinct from $u, v$. The graph $G^{\prime}$ obtained from $G$ by performing the same reduction operation will then be $\mathcal{M}_{l c}$-connected since it is the 2-sum of $G_{1}$ and $G_{2}^{\prime}$ along $u v$.

Figure 8 provides an illustration of Theorem 6.13. In this figure a thick edge or loop means the next step in the construction will be a 1-extension which deletes that edge or loop, and a dashed edge or loop means the next step in the construction will add that edge or loop.

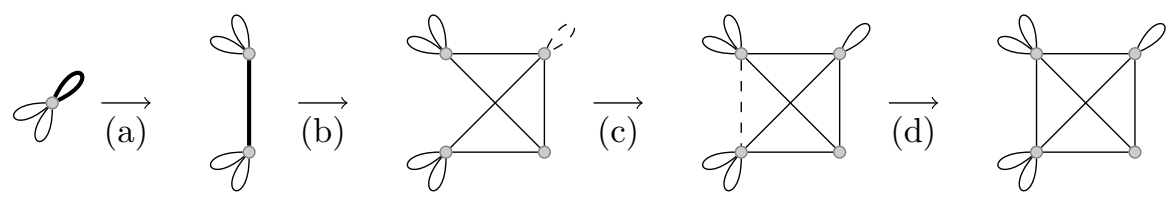

FiguRE 8. A construction of the rigid $\mathcal{M}_{l c}$-connected graph drawn on the far right from a copy of $K_{1}^{[3]}$, by (a) 1 -extension on a loop, (b) $K_{4}$-extension, (c) loop addition and (d) edge addition.

Theorem 6.13 implies in particular that all rigid $\mathcal{M}_{l c}$-circuits can be constructed from $K_{1}^{[3]}$ by applying the operations of 1 -extension, $K_{4}$-extension and adding a new edge or loop. Since every rigid $\mathcal{M}_{l c}$-circuit $G=(V, E, L)$ satisfies $|E|+|L|=2|V|$ we can never use the edge/loop addition operation when constructing a rigid circuit from $K_{1}^{[3]}$. This gives the following result.

Theorem 6.14. A looped simple graph is a rigid $\mathcal{M}_{l c}$-circuit if and only if it can be obtained from $K_{1}^{[3]}$ by recursively applying the 1 -extension and $K_{4}$-extension operations.

\section{BALANCED $\mathcal{M}_{l c}$-CONNECTED GRAPHS}

We next consider reductions that preserve balance as well as $\mathcal{M}_{l c}$-connectivity. We will need two more structural results in unbalanced separations.

Lemma 7.1. Let $G$ be $\mathcal{M}_{l c}$-connected and let $\{u, v\}$ be an unbalanced 2-separator in $G$. Then $u$ and $v$ each have at least four incident edges or loops in $G$. Furthermore, if $G^{\prime}$ 
is obtained from $G$ by performing an edge/loop deletion or 1-reduction, then $\{u, v\}$ is an unbalanced 2-separator in $G^{\prime}$.

Proof. Let $\left(G_{1}, G_{2}\right)$ be an unbalanced 2-separation in $G$ with $V\left(G_{1}\right) \cap V\left(G_{2}\right)=\{u, v\}$. Then $G_{1}+u v$ and $G_{2}+u v$ are $\mathcal{M}_{l c}$-connected by Lemma 6.12, Hence $u$ and $v$ are each incident with at least three edges or loops in $G_{i}+u v$ for $i=1,2$. This implies that $u$ and $v$ each have at least four incident edges or loops in $G$. In particular, neither $u$ nor $v$ is a node of $G$. It is now straightforward to check that $\{u, v\}$ is an unbalanced 2-separator in $G^{\prime}$.

Our next lemma shows that a rigid looped simple graph cannot contain two 'crossing' unbalanced 2-separations.

Lemma 7.2. Supose that $G=(V, E, L)$ is rigid looped simple graph and $\left(G_{1}, G_{2}\right),\left(G_{1}^{\prime}, G_{2}^{\prime}\right)$ are two unbalanced 2-separations in $G$ with $G_{i}=\left(V_{i}, E_{i}, L_{i}\right)$ and $G_{i}^{\prime}=\left(V_{i}^{\prime}, E_{i}^{\prime}, L_{i}^{\prime}\right)$ for $i=1,2, L_{2}=\emptyset=L_{2}^{\prime}, V_{1} \cap V_{2}=\{u, v\}, V_{1}^{\prime} \cap V_{2}^{\prime}=\left\{u^{\prime}, v^{\prime}\right\}$ and $\{u, v\} \cap\left\{u^{\prime}, v^{\prime}\right\}=\emptyset$. Then $\left\{u^{\prime}, v^{\prime}\right\} \subseteq V_{i}$ for some $i \in\{1,2\}$.

Proof. Suppose for a contradiction that $u^{\prime} \in V_{1}$ and $v^{\prime} \in V_{2}$. If $\{u, v\} \subseteq V_{i}^{\prime}$ for some $i \in\{1,2\}$ then either $G-u^{\prime}$ or $G-v^{\prime}$ would have a loopless component. This would contradict the hypothesis that $G$ is rigid and hence we may assume that $u \in V_{1}^{\prime}$ and $v \in V_{2}^{\prime}$.

Let $H_{1}=G_{1}\left[V_{1} \cap V_{1}^{\prime}\right], H_{2}=G_{2}\left[V_{1}^{\prime} \cap V_{2}\right], H_{3}=G_{2}\left[V_{2} \cap V_{2}^{\prime}\right], H_{4}=G_{2}^{\prime}\left[V_{1} \cap V_{2}^{\prime}\right]$, and put $n_{i}=\left|V\left(H_{i}\right)\right|$ for $1 \leq i \leq 4$, see Figure 9. Then $H_{1}, H_{2}, H_{3}, H_{4}$ cover $E \cup L$ and $H_{2}, H_{3}, H_{4}$ are loopless. This gives

$$
r(G) \leq \sum_{i=1}^{4} r\left(H_{i}\right) \leq 2 n_{1}+\sum_{i=2}^{4}\left(2 n_{i}-3\right)=2|V|-1
$$

and again contradicts the hypothesis that $G$ is rigid.

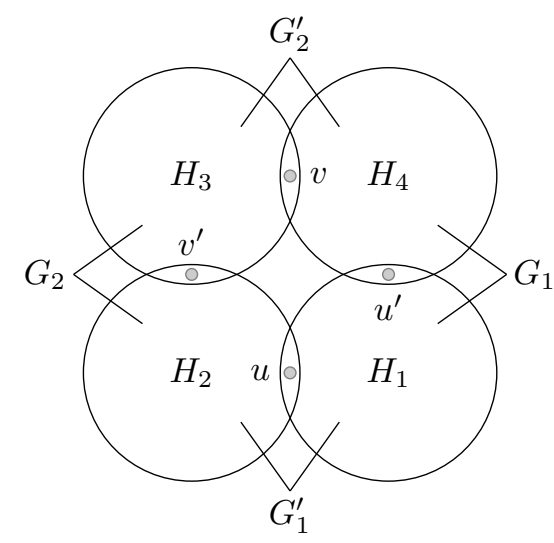

Figure 9. The subgraphs $H_{1}, H_{2}, H_{3}, H_{4}$ in the proof of Lemma 7.2 .

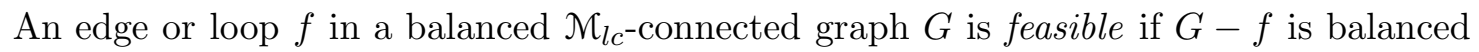
and $\mathcal{M}_{l c}$-connected, and a node $v$ is feasible if there exists a 1-reduction at $v$ which results in a balanced $\mathcal{M}_{l c}$-connected graph. Figure 10 illustrates the difference between admissibility and feasibility. On the far left, the vertex $x$ is not admissible in $H$. Each 1-reduction at $x$ that adds a loop creates a vertex of degree 2, and the 1-reduction at $x$ which adds an edge results in a graph with only 3 loops, none of which is contained in an $\mathcal{M}_{l c}$-circuit. The only admisible 1-reduction at the vertex $y$ in the graph $G$ is the one that adds the edge $y_{1} y_{2}$. 
However, the graph $G-y+y_{1} y_{2}$ is not balanced since $\left(G-y+y_{1} y_{2}\right)-\{u, v\}$ has no loops. Therefore $y$ is admisible but not feasible in $G$. The 1-reduction at $y_{1}$ which adds the edge $v y$ results in a balanced rigid $\mathcal{M}_{l c}$-circuit, so the vertex $y_{1}$ is feasible in $G$.

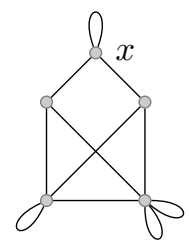

$H$

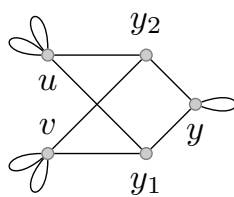

$G$

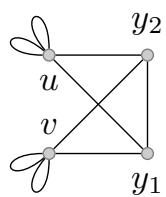

$G-y+y_{1} y_{2}$

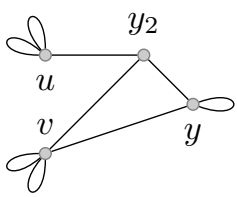

$G-y_{1}+v y$

Figure 10. The vertex $x$ is non-admissible in $H$, the vertex $y$ is admissible but non-feasible in $G$, and the vertex $y_{1}$ is feasible in $G$.

Theorem 7.3. Let $G=(V, E, L)$ be a balanced, $\mathcal{M}_{l c}$-connected looped simple graph distinct from $K_{1}^{[3]}$. Then some edge, loop, or node of $G$ is feasible.

Proof. Suppose, for a contradiction, that all possible edge/loop deletions and node 1reductions of $G$ fail to be either $\mathcal{M}_{l c}$-connected or balanced. Theorem 6.6 implies that $G$ contains either an admissible node $w$ or an admissible edge or loop $f$. By assumption neither $w$ nor $f$ is feasible. Let $G^{\prime}$ be the result of deleting $f$ or performing an admissible 1-reduction at $w$. Then $G^{\prime}$ is $\mathcal{N}_{l c}$-connected and not balanced. Hence $G^{\prime}$ contains an unbalanced 2-separation $H_{1}, H_{2}$ where $H_{1}$ is loopless and $V\left(H_{1}\right) \cap V\left(H_{2}\right)=\{u, v\}$. We may suppose that the pair $(r,\{u, v\})$, where $r \in\{w, f\}$, has been chosen so that $X=V\left(H_{1}\right) \backslash\{u, v\}$ is as small as possible.

Let $H_{1}^{+}=H_{1}+u v$ and $H_{2}^{+}=H_{2}+u v$.

Claim 7.4. $\mathrm{H}_{1}^{+}$and $\mathrm{H}_{2}^{+}$are $\mathcal{M}_{l c}$-connected and $\mathrm{H}_{2}^{+}$is redundantly rigid.

Proof of Claim. Corollary 6.3 implies that $H_{1}^{+}$and $H_{2}^{+}$are $\mathcal{M}_{l c}$-connected. Corollary 6.3 now implies that $\mathrm{H}_{2}^{+}$is redundantly rigid.

Note that the minimality of $X$ implies that $H_{1}^{+}$is 3-connected.

Claim 7.5. $u v \notin E$.

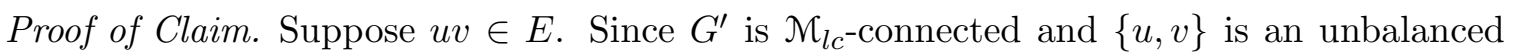
2 -separator in $G^{\prime}$, Lemma 6.12 implies that $G^{\prime}-u v$ is $\mathcal{M}_{l c}$-connected. Since $G-u v$ is obtained from $G^{\prime}-u v$ by an edge addition or a 1-extension, $G-u v$ is $\mathcal{M}_{l c}$-connected by Lemma 6.5. It remains to show that $G-u v$ is balanced.

Suppose $\left\{u^{\prime}, v^{\prime}\right\}$ is an unbalanced 2-separator in $G-u v$. Since $G$ is balanced, $u$ and $v$ belong to different components of $(G-u v)-\left\{u^{\prime}, v^{\prime}\right\}$, at least one of which is loopless. Furthermore, $\left\{u^{\prime}, v^{\prime}\right\}$ is an unbalanced 2-separator in $G^{\prime}-u v$ by Lemma 7.1. This implies that $G^{\prime}-u v$ does not contain three internally disjoint $u v$-paths. Since $H_{1}^{+}$is 3-connected, there are two internally disjoint $u v$-paths in $H_{1}^{+}-u v$. Hence $u^{\prime}, v^{\prime} \in X$ and $u$ and $v$ belong to different components $J_{u}$ and $J_{v}$ of $H_{2}^{+}-u v$, see Figure 11 for an illustration. Note that $J_{u}$ and $J_{v}$ both contain loops since $G^{\prime}$ is rigid. This contradicts the fact that either $u$ or $v$ belongs to a loopless component of $(G-u v)-\left\{u^{\prime}, v^{\prime}\right\}$ since $G-u v$ is obtained from $G^{\prime}-u v$ by applying a 1-extension or edge/loop addition. 


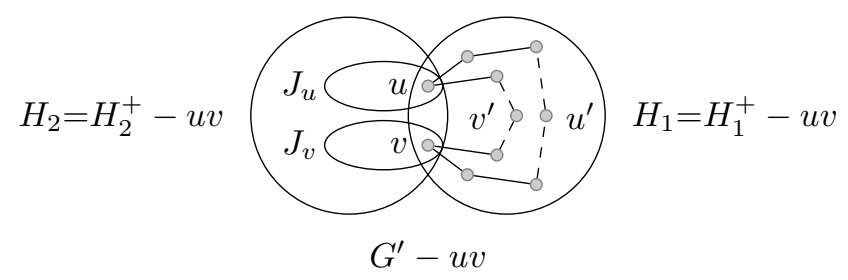

Figure 11. An illustration of the structure of $G^{\prime}-u v$ in the proof of Claim 7.5. The 3-connectivity of $H_{1}^{+}$implies there are two disjoint $u v$-paths within $H_{1}=H_{1}^{+}-u v$, and hence removing $u^{\prime}$ and $v^{\prime}$ must destroy these paths.

Our strategy in the remainder of the proof is to show that some edge or node of $G$ in $H_{1}$ is feasible in $G$. We have to be careful when considering the edges and nodes of $H_{1}$ since not all of them are edges or nodes in $G$. In addition a vertex which is a node in both $H_{1}$ and $G$ may be incident with different edges in each graph. We use the following notation to handle this. We first put $E^{\dagger}\left(H_{1}\right)=E\left(H_{1}\right) \cap E(G)$. If $r=w$, we let $V^{\dagger}\left(H_{1}\right)=X-N_{G}(w)$ and, if $r=f$ and $f=y z$, then we let $V^{\dagger}\left(H_{1}\right)=X-\{y, z\}$. If the reduction operation which converts $G$ to $G^{\prime}$ adds an edge $e$ between two vertices of $H_{1}$ we put $\theta=e$. Otherwise there is a unique vertex $x$ of $X$ which is incident/adjacent to $r$ and we put $\theta=x$.

Claim 7.6. Suppose that $G-e$ is $\mathcal{M}_{l c}$-connected for some $e \in E^{\dagger}\left(H_{1}\right)$. Then $H_{1}-\{u, v, e\}$ is connected.

Proof of Claim. Suppose $H_{1}-\{u, v, e\}$ has two components $J_{1}, J_{2}$. Choose $i \in\{1,2\}$ such that $\theta \notin V\left(J_{i}\right) \cup E\left(J_{i}\right)$. Then $\{u, v\}$ is an unbalanced 2-separation of $G-e$ with $J_{i}$ as a component and $V\left(J_{i}\right)$ is properly contained in $X$. This contradicts the minimality of $X$.

Claim 7.7. $G-e$ is not $\mathcal{M}_{l c}$-connected for all $e \in E^{\dagger}\left(H_{1}\right)$.

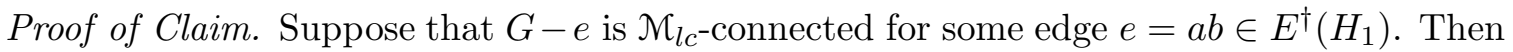
$G-e$ is not balanced so there exists an unbalanced 2-separator $T$ in $G-e$. Since $G$ is balanced, $a$ and $b$ are in different components of $(G-e)-T$.

We can apply Lemma 7.1 to $G-e$ and $G^{\prime}$ respectively to deduce that $T$ and $S=\{u, v\}$ are unbalanced 2-separators in $G^{\prime \prime}=G^{\prime}-e$. Since $G^{\prime}$ is $\mathcal{M}_{l c}$-connected and contains a loop, it is redundantly rigid by Corollary 6.3. Hence $G^{\prime \prime}=G^{\prime}-e$ is rigid. Lemma 7.2 now implies that $T \subseteq V\left(H_{i}\right)$ for some $i \in\{1,2\}$. Since $H_{1}-\{u, v, e\}$ is connected by Claim [7.6, $G^{\prime \prime}[X]$ is a component of $G^{\prime \prime}-S$. Since $a, b \in X \cup S$ and $T$ separates $a, b$ in $G-e$, we have $T \cap X \neq \emptyset$. Hence $T \subseteq V\left(H_{1}\right)$.

Let $X_{T}$ be the vertex set of a loopless component of $(G-e)-T$. Since $G$ is balanced, each component of $H_{2}-S$ contains a loop and hence $X_{T} \cap\left(V\left(H_{2}-S\right)=\emptyset\right.$. This implies that $X_{T} \cup T \subseteq X \cup S \cup\{r\}$ so we may contradict the minimality of $X$ by showing that $r$ and some vertex of $X \cup S$ does not belong to $X_{T} \cup T$. We know that $r$ is adjacent/incident to some vertex $x \in X$. If $r$ is also adjacent/incident to some vertex of $V\left(H_{2}\right) \backslash S$ then the facts that $r$ does not belong to $G^{\prime}-e$ and $T$ is an unbalanced 2-separator of $G-e$ gives $r, x \notin X_{T} \cup T$. Similarly, if $r$ is only adjacent/incident to vertices of $H_{1}$, then either $r$ is a loop or $r$ is a node incident with a loop and we again have $r, x \notin X_{T} \cup T$.

Claim 7.8. $H_{1}^{+}-e$ is not $\mathcal{M}_{l c}$-connected for all $e \in E^{\dagger}\left(H_{1}\right)$. 
Proof of Claim. Suppose $H_{1}^{+}-e$ is $\mathcal{M}_{l c}$-connected. Then $G^{\prime}-e$ is the 2 -sum of $H_{1}^{+}-e$

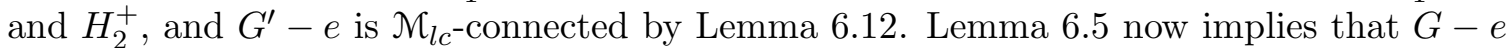
is $\mathcal{M}_{l c}$-connected, contradicting Claim 7.7 .

Claim 7.9. Suppose $p \in V^{\dagger}\left(H_{1}\right)$ is a node of $H_{1}^{+}, N(p)=\{q, s, t\}$ and $G-p+$ st is $\mathcal{M}_{l c}$-connected. Then $\left(H_{1}-p+s t\right)-\{u, v\}$ is connected.

Proof of Claim. Suppose $\left(H_{1}-p+s t\right)-\{u, v\}$ has two components $J_{1}, J_{2}$. Choose $i \in\{1,2\}$ such that $\theta \notin V\left(J_{i}\right) \cup E\left(J_{i}\right)$. Then $\{u, v\}$ is an unbalanced 2-separation in $G-p+s t$ and $J_{i}$ is a loopless component of $(G-p+s t)-\{u, v\}$. This contradicts the minimality of $X$.

Claim 7.10. Every node $p$ of $H_{1}^{+}$in $V^{\dagger}\left(H_{1}\right)$ is non-admissible in $G$.

Proof of Claim. Suppose $p \in V^{\dagger}\left(H_{1}\right)$ is a node of $H_{1}^{+}$with $N(p)=\{q, s, t\}$ and $G-p+s t$ is $\mathcal{M}_{l c}$-connected. Then $G-p+s t$ is not balanced, so has an unbalanced 2-separator $T$. Since $G$ is balanced, st and $q$ are in different components of $(G-p+s t)-T$.

We can apply Lemma 7.1 to $G-p+s t$ and $G^{\prime}$ respectively to deduce that $T$ and $S=\{u, v\}$ are unbalanced 2-separators in $G^{\prime \prime}=G^{\prime}-p+s t$. Since $G^{\prime}$ is $\mathcal{M}_{l c^{-c o n n e c t e d ~ a n d ~ c o n t a i n s ~}}$ loops it is redundantly rigid by Corollary 6.3. Hence $G^{\prime}-p q$ is rigid. Since $G^{\prime}-p$ is obtained from $G^{\prime}-p q$ by deleting a vertex with two incident edges, it is rigid. Since $G^{\prime \prime}$ is obtained from $G^{\prime}-p$ by an edge addition, it is also rigid. Lemma 7.2 now implies that $T \subseteq V\left(H_{i}\right)$ for some $i \in\{1,2\}$. Since $H_{1}-p+s t-\{u, v\}$ is connected by Claim [7.6, $G^{\prime \prime}[X-p]$ is a component of $G^{\prime \prime}-S$. Since $q, s, t \in X \cup S$ and $T$ separates st from $q$ in $G^{\prime \prime}$, we have $T \cap X \neq \emptyset$. Hence $T \subseteq V\left(H_{1}\right)$.

Let $X_{T}$ be the vertex set of a loopless component of $(G-p+s t)-T$. Since $G$ is balanced, each component of $H_{2}-S$ contains a loop and hence $X_{T} \cap\left(V\left(H_{2}\right)-S\right)=\emptyset$. This implies that $X_{T} \cup T \subseteq(X \cup S)+r-p$ so we may contradict the minimality of $X$ by showing that $r$ does not belong to $X_{T} \cup T$. This is trivially true if $r=f$, so we may assume $r=w$. We know that $w$ is adjacent to some vertex $x \in X$. If $w$ is also adjacent to some vertex of $H_{2}-S$ then the facts that $w$ does not belong to $G^{\prime}-p+s t$ and $T$ is an unbalanced 2 -separator of $G-p+s t$ give $w \notin X_{T} \cup T$. Similarly, if $w$ is only adjacent to vertices of $H_{1}$, then $w$ is a node incident with a loop and we again have $w \notin X_{T} \cup T$.

Claim 7.11. Every node $p$ of $H_{1}^{+}$in $V^{\dagger}\left(H_{1}\right)$ is non-admissible in $H_{1}^{+}$.

Proof of Claim. Suppose $p$ is a node with $N(p)=\{q, s, t\}$ and $H_{1}^{+}-p+s t$ is $\mathcal{M}_{l c^{-c o n n e c t e d}}$. Then $G^{\prime}-p+s t$ is the 2-sum of $H_{1}^{+}-p+s t$ and $H_{2}^{+}$and $G^{\prime}-p+s t$ is $\mathcal{M}_{l c}$-connected by Lemma 6.12, It follows from Lemma 6.5 that $G-p+s t$ is $\mathcal{M}_{l c}$-connected, contradicting Claim 7.10

Claim 7.12. $H_{1}^{+}$is an $\mathcal{M}_{l c}$-circuit.

Proof of Claim. This follows immediately from Theorem 6.11(b), Claims 7.4, 7.8 and 7.11, and the definition of $V^{\dagger}\left(H_{1}\right)$.

Claim 7.13. $H_{1}^{+}$is isomorphic to $K_{4}$.

Proof of Claim. Suppose $H_{1}^{+}$is not isomorphic to $K_{4}$. By Claim 7.11, no node of $H_{1}^{+}$in $V^{\dagger}\left(H_{1}\right)$ is admissible in $H_{1}^{+}$. Theorem 6.11(a), Claim 7.12 and the definition of $V^{\dagger}\left(H_{1}\right)$ now imply that $G^{\prime}=G-w+x y$, for some $x, y \in V\left(H_{1}\right)$ and $u, v, x, y$ are the only admissible nodes in $H_{1}^{+}$. We shall show that $x$ is a feasible node in $G$.

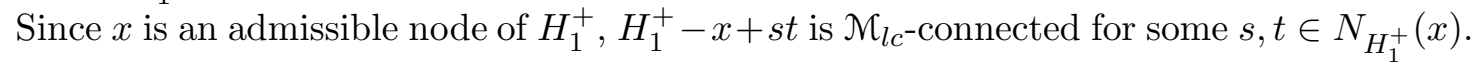
Let $N_{H_{1}^{+}}(x)=\{q, s, t\}$. Since $x y$ is an edge of $H_{1}^{+}$and $y$ is a node of $H_{1}^{+}$, we must have 
$y \in\{s, t\}$. Without loss of generality suppose $y=t$. Since $G^{\prime}-x+s y$ is the 2 -sum of $H_{1}^{+}-x+s y$ and $H_{2}^{+}$, and $H_{2}^{+}$is $\mathcal{M}_{l c}$-connected by Claim [7.4 Lemma 6.12 implies that $G^{\prime}-x+s y$ is $\mathcal{M}_{l c}$-connected. Since $G-x+s w$ is a 1-extension of $G^{\prime}-x+s y$, Lemma 6.5 now implies that $G-x+s w$ is $\mathcal{M}_{l c}$-connected.

Suppose $H_{1}^{+}-x+s y-\{u, v\}$ is disconnected. Since $H_{1}^{+}$is 3-connected, $H_{1}^{+}-x+s y-\{u, v\}$ has two components $J_{1}, J_{2}$ with $s y \in E\left(J_{1}\right)$ and $q \in V\left(J_{2}\right)$. Then $V\left(J_{2}\right)$ is properly contained in $X$. Since $J_{2}$ is a loopless component of $(G-x+s w)-\{u, v\}$, this contradicts the minimality of $X$. Thus $H_{1}^{+}-x+s y-\{u, v\}$ is connected.

Since $x$ is not feasible in $G, G-x+s w$ is not balanced. Let $T$ be an unbalanced 2separator in $G-x+s w$. Since $G$ is balanced, $T$ separates $s w$ and $q$. We can apply Lemma 7.1 to $G-x+s w$ and $G^{\prime}$ respectively to deduce that $T$ and $S=\{u, v\}$ are unbalanced 2-separators in $G^{\prime \prime}=G^{\prime}-x+s y$. Since $G^{\prime}$ is $\mathcal{M}_{l c^{-}}$-connected and contains a loop, Corollary 6.3 implies that $G^{\prime}$ is redundantly rigid, and hence $G^{\prime}-x q$ is rigid. Since $G^{\prime}-x$ is obtained from $G^{\prime}-x q$ by deleting a vertex with two incident edges, it is rigid. Since $G^{\prime \prime}=G^{\prime}-x+s y$ is obtained from $G^{\prime}-x$ by an edge addition, it is also rigid. Lemma 7.2 now implies that $T \subseteq V\left(H_{i}\right)$ for some $i \in\{1,2\}$. Since $\left(H_{1}^{+}-x+s y\right)-\{u, v\}$ is connected, $G^{\prime \prime}[X-x]$ is a component of $G^{\prime \prime}-S$. Since $q, s, y \in X \cup S$, and $T$ separates $s y$ and $q$ in $G^{\prime \prime}$, we have $T \cap X \neq \emptyset$. Hence $T \subseteq V\left(H_{1}\right)$.

Let $X_{T}$ be the vertex set of the component of $(G-x+s w)-T$ which is loopless. Since $G$ is balanced, each component of $H_{2}^{+}-S$ contains a loop and hence $X_{T} \cap\left(V\left(H_{2}\right)-S\right)=\emptyset$. This implies that $X_{T} \cup T \subseteq(X \cup S)+w-x$, so we may contradict the minimality of $X$ by showing that $w$ does not belong to $X_{T} \cup T$. Note that $w \notin T$ by Lemma 7.1, If $w$ is adjacent to some vertex $z \in V\left(H_{2}\right) \backslash S$ then $z \notin X_{T} \cup T$. Since $z w \in E(G-x+s w)$ and $T$ is an unbalanced 2-separator, $w \notin X_{T}$. Similarly, if $w$ is only adjacent to vertices of $H_{1}^{+}$, then $w$ is a node incident with a loop and we again have $w \notin X_{T}$.

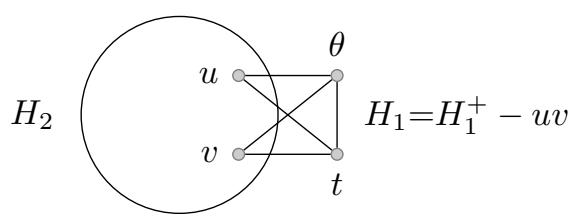

Figure 12. The structure of $G^{\prime}$ in the proof of Claim 7.14,

Claim 7.14. $G^{\prime}=G-w+x y$ for some $x, y \in V\left(H_{1}\right)$ and hence $\theta=x y \in E\left(H_{1}\right)$.

Proof of Claim. Suppose that the claim is false. Then $\theta$ is a vertex in $X, V\left(H_{1}\right)=\{u, v, \theta, t\}$ and $t$ is a node of $G$, see Figure 12. We will show that $G-t+u v$ is balanced and $\mathcal{M}_{l c^{-}}$ connected. Note that $u v \notin E$ by Claim [7.5. Note also that $G-t+u v$ can be obtained from $\mathrm{H}_{2}^{+}$by either a sequence of one 1-extension and one edge addition (in the case that $G^{\prime}=G-f$ ) or two 1-extensions (in the case when $G^{\prime}=G-w+z_{1} z_{2}$ for some $z_{1}, z_{2} \notin X$ ). Since $H_{2}^{+}$is $\mathcal{M}_{l c}$-connected by Claim [7.4, it follows from Lemma 6.5 that $G-t+u v$ is $\mathcal{M}_{l c}$-connected. Since $\theta$ is adjacent to $u$ and $v$ and $G$ is balanced there is no unbalanced 2 -separation separating $\theta$ from $u v$ in $G-t+u v$. Thus $G-t+u v$ is balanced.

Claim 7.15. $X \neq\{x, y\}$.

Proof of Claim. Suppose that $X=\{x, y\}$. Then $x, y$ are nodes of $G$. We shall show that $G-x+w v$ is $\mathcal{M}_{l c}$-connected and balanced. Note that $w v \notin E$ since, if $w$ has a neighbour $z$ 
distinct from $x, y$, then $z \in V\left(H_{2}\right) \backslash S$. Note further that $G-x+w v$ can be obtained from $\mathrm{H}_{2}^{+}$by a sequence of two 1-extensions. Since $H_{2}^{+}$is $\mathcal{M}_{l c}$-connected by Claim 7.4, Lemma 6.5 implies that $G-x+w v$ is $\mathcal{M}_{l c}$-connected. Suppose that $G-x+w v$ is not balanced. Since $G$ is balanced, there is an unbalanced 2-separator $T$ in $G-x+w v$ that separates $u$ and $w v$. Since $u, w$ and $v$ are all neighbours of $y$ in $G-x+w v$, we must have $y \in T$. Since $y$ is a node in $G-x+w v$, this contradicts Lemma 7.1. Thus $G-x+w v$ is balanced.

We can now complete the proof of the theorem. Claims 7.14 and 7.15 allow us to assume, after relabelling if necessary, that $X=\{x, t\}$ and $v=y$. Thus $x$ is a node of $G$. We will show that $G-x+w t$ is $\mathcal{M}_{l c}$-connected and balanced. Note that $w t \notin E$ since the neighbour of $w$ distinct from $x, y$ belongs to $V-(X \cup S)$. Note further that $G-x+w t$ can be obtained from $H_{2}^{+}$by a sequence of two 1-extensions. Since $H_{2}^{+}$is $\mathcal{M}_{l c}$-connected by Claim 7.4, it follows from Lemma 6.5 that $G-x+w t$ is $\mathcal{M}_{l c}$-connected. Suppose that $G-x+w t$ is not balanced. Since $G$ is balanced there is an unbalanced 2-separator $T$ in $G-x+w t$ separating $u$ and $w t$. Since $u t$ is an edge of $G-x+w t$ we must have $t \in T$. Since $t$ is a node in $G-x+w t$, this contradicts Lemma 7.1. Thus $G-x+w v$ is balanced.

Lemma 6.5 and Lemma 7.1 imply that the operations of edge/loop addition and 1extension preserve the properties of being $\mathcal{M}_{l c}$-connected and balanced. Combined with Theorem 7.3, this immediately gives the following recursive construction.

Theorem 7.16. A looped simple graph is balanced and $\mathcal{M}_{l c}$-connected if and only if it can be obtained from $K_{1}^{[3]}$ by recursively applying the operations of performing a 1-extension and adding a new edge or loop.

Consider the balanced, $\mathcal{M}_{l c^{-}}$-connected graph $G$ drawn on the far right in Figure 13, We gave a construction of $G$ from $K_{1}^{[3]}$ in Figure [8. However, the second step in this construction, where we use $K_{4}$-extension, resulted in an unbalanced graph. In Figure 13, we show that we can obtain $G$ from $K_{1}^{[3]}$ by using only 1-extensions and edge or loop additions.

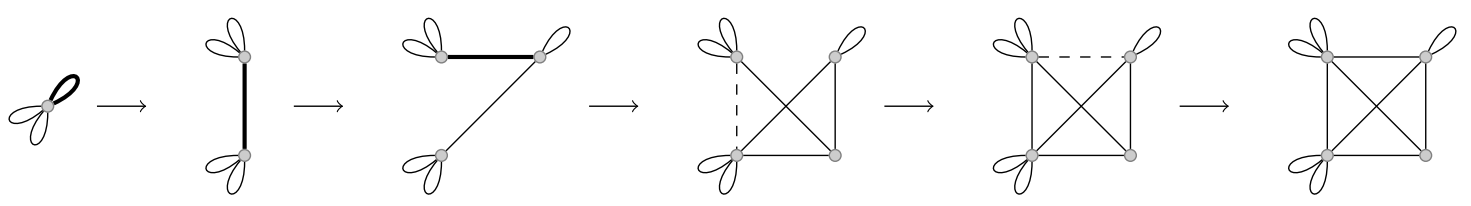

FIgURE 13. An illustration of the recursive construction given in Theorem 7.16,

\section{Global Rigidity}

We will use our recursive construction to characterise generic global rigidity. We first need a lemma which shows that the properties of redundant rigidity and $\mathcal{M}_{l c}$-connectedness are equivalent for connected balanced graphs.

Lemma 8.1. Let $G$ be a balanced looped simple graph. Then $G$ is $\mathcal{M}_{l c}$-connected if and only if $G$ is connected and redundantly rigid.

Proof. Necessity follows from Corollary 6.3 and the assumption that $G$ is $\mathcal{M}_{l c}$-connected.

To prove sufficiency, we suppose, for a contradiction that $G$ is connected and redundantly rigid but not $\mathcal{M}_{l c}$-connected. Let $H_{1}, H_{2}, \ldots, H_{m}$ be the $\mathcal{M}_{l c}$-components of $G$. Let $V_{i}=$ 
$V\left(H_{i}\right), X_{i}=V_{i} \backslash \bigcup_{j \neq i} V_{j}$ and $Y_{i}=V_{i} \backslash X_{i}$. Since $G$ is connected, $\left|Y_{i}\right| \geq 1$, and since $G$ is balanced, $\left|Y_{i}\right| \geq 3$ when $H_{i}$ is loopless.

We may assume that $H_{1}, H_{2}, \ldots, H_{s}$ are loopless and $H_{s+1}, H_{s+2}, \ldots, H_{m}$ are not. Then

$$
\begin{aligned}
r(G) & =\sum_{i=1}^{s}\left(2\left|V_{i}\right|-3\right)+\sum_{i=s+1}^{m} 2\left|V_{i}\right| \\
& =\sum_{i=1}^{s}\left(2\left|X_{i}\right|+2\left|Y_{i}\right|-3\right)+\sum_{i=s+1}^{m}\left(2\left|X_{i}\right|+2\left|Y_{i}\right|\right) \\
& \geq \sum_{i=1}^{m}\left(2\left|X_{i}\right|+\left|Y_{i}\right|\right)+m-s,
\end{aligned}
$$

where the final inequality follows from the fact that $\left|Y_{i}\right| \geq 3$ for $1 \leq i \leq s$ and $\left|Y_{i}\right| \geq 1$ for $s+1 \leq i \leq m$. Since the $X_{i}$ are disjoint we have $\sum_{i=1}^{m}\left|X_{i}\right|=\left|\bigcup_{i=1}^{m} X_{i}\right|$. Also each element of $Y_{i}$ is contained in at least one other $Y_{j}$ with $j \neq i$. Hence we have $\sum_{i=1}^{m}\left|Y_{i}\right| \geq 2\left|\bigcup_{i=1}^{m} Y_{i}\right|$. In addition the hypothesis that $G$ is balanced implies that at least one $H_{i}$ contains a loop so $m>s$. Hence

$$
r(G) \geq 2\left(\left|\bigcup_{i=1}^{m} X_{i}\right|+\left|\bigcup_{i=1}^{m} Y_{i}\right|\right)+m-s>2|V(G)| .
$$

This contradicts the fact that $r(G) \leq 2|V(G)|$.

We may use Lemma 8.1 to restate Theorem 7.16 as

Theorem 8.2. A looped simple graph is balanced, connected and redundantly rigid if and only if it can be obtained from $K_{1}^{[3]}$ by recursively applying the operations of performing a 1-extension and adding a new edge or loop.

We can now characterise global rigidity for 2-dimensional generic linearly constrained frameworks.

Theorem 8.3. Suppose $G$ is a connected looped simple graph with at least two vertices and $(G, p, q)$ is a generic realisation of $G$ as a linearly constrained framework in $\mathbb{R}^{2}$. Then the following statements are equivalent:

(a) $(G, p, q)$ is globally rigid;

(b) $G$ is balanced and redundantly rigid;

(c) $(G, p, q)$ has a full rank equilibrium stress.

Proof. The implications (a) $\Rightarrow$ (b) and (c) $\Rightarrow$ (a) follow from Theorems 3.2 and 4.5 , respectively. It remains to prove that (b) $\Rightarrow$ (c). We use induction on the number of vertices of $G$ to show that $(G, p, q)$ has a full rank equilibrium stress whenever $G$ is connected, balanced and redundantly rigid. It is straighforward to check that every generic realisation of the smallest redundantly rigid looped simple graph $K_{1}^{[3]}$ has a full rank equilibrum stress (given by a $1 \times 1$ stress matrix of rank zero). The induction step now follows by using Lemma 4.6, and Theorems 4.7 and 8.2 .

We can use Theorem 8.3 and the fact that a linearly constrained framework is globally rigid if and only if each of its connected components is globally rigid to deduce:

Theorem 8.4. Suppose $(G, p, q)$ is a generic linearly constrained framework in $\mathbb{R}^{2}$. Then $(G, p, q)$ is globally rigid if and only if $G$ is balanced and each connected component of $G$ is either a single vertex with two loops or is redundantly rigid. 
Theorem 2.1 implies that redundant rigidity can be checked efficiently by graph orientation or pebble game type algorithms [2, 19]. Since we can also check the property of being balanced in polynomial time, Theorem 8.4 gives rise to an efficient algorithm to decide whether a given looped simple graph is generically globally rigid in $\mathbb{R}^{2}$.

We conclude this section by mentioning a possible direction for future research. As mentioned in the introduction, [8] gives a characterisation of generic rigidity for linearly constrained frameworks in $\mathbb{R}^{d}$ when each vertex is constrained to lie in an affine subspace of sufficiently small dimension compared to $d$. It would be interesting to obtain an analogous characterisation for generic global rigidity.

\section{The NUMBER OF EQUiVAlENT REALISATIONS}

We will extend Theorem 8.4 by determining the number of distinct frameworks which are equivalent to a given generic linearly constrained framework $(G, p, q)$ when $G=(V, E, L)$ is a rigid $\mathcal{M}_{l c}$-connected looped simple graph. For $u, v \in V$, let $b(u, v)$ be the number of loopless connected components of $G-\{u, v\}$ and put $b(G)=\sum_{u, v \in V} b(u, v)$.

Theorem 9.1. Suppose $(G, p, q)$ is a generic linearly constrained framework in $\mathbb{R}^{2}$ and that $G$ is rigid and $\mathcal{M}_{l c}$-connected. Then there are exactly $2^{b(G)}$ distinct frameworks which are equivalent to $(G, p, q)$.

Our proof of Theorem 9.1 is similar to the proof of an analogous result for bar-joint frameworks [15, Theorem 8.2]. We will indicate below how the latter can be adapted to prove Theorem 9.1 .

We first need a result on generic points in $\mathbb{R}^{n}$. We will denote the algebraic closure of a field $\mathbb{K}$ by $\overline{\mathbb{K}}$.

Lemma 9.2. Let $f: \mathbb{R}^{n} \rightarrow \mathbb{R}^{n}$ by $f(\mathbf{x})=\left(f_{1}(\mathbf{x}), f_{2}(\mathbf{x}), \ldots, f_{n}(\mathbf{x})\right)$, where $f_{i}(\mathbf{x})$ is a polynomial with coefficients in some extension field $\mathbb{K}$ of $\mathbb{Q}$ for all $1 \leq i \leq n$. Suppose that $\max _{\mathbf{x} \in \mathbb{R}^{n}}\left\{\right.$ rank $\left.\left.d f\right|_{\mathbf{x}}\right\}=n$. If either $\mathbf{x}$ or $f(\mathbf{x})$ is generic over $\mathbb{K}$, then $\mathbf{x}$ and $f(\mathbf{x})$ are both generic over $\mathbb{K}$ and $\overline{\mathbb{K}(\mathbf{x})}=\overline{\mathbb{K}(f(\mathbf{x}))}$.

The proof of Lemma 9.2 is the same as that for [15, Lemmas 3.1, 3.2]. The only difference being that we work with polynomials with coefficients in $\mathbb{K}$ rather than $\mathbb{Z}$.

Let $G=(V, E, L)$ be a looped simple graph with $E=\left\{e_{1}, e_{2}, \ldots, e_{m}\right\}, F=\left\{\ell_{1}, \ell_{2}, \ldots, \ell_{s}\right\}$ and $q: L \rightarrow \mathbb{R}^{2}$. The rigidity map $f_{G, q}: \mathbb{R}^{2|V|} \rightarrow \mathbb{R}^{|E \cup L|}$ is defined by putting

$$
f_{G, q}(p)=\left(f_{e_{1}}(p), \ldots, f_{e_{m}}(p), f_{\ell_{1}}(p) \ldots, f_{\ell_{s}}(p)\right)
$$

where $f_{e_{i}}(p)=\|p(u)-p(v)\|^{2}$ when $e_{i}=u v$ and $f_{\ell_{j}}(p)=q\left(\ell_{j}\right) \cdot p(v)$ when $\ell_{j}$ is incident to $v$.

Lemma 9.3. Let $(G, p, q)$ be a generic, rigid, linearly constrained framework in $\mathbb{R}^{2}$ and suppose that $\left(G, p^{\prime}, q\right)$ is an equivalent framework. Then $\overline{\mathbb{Q}(p, q)}=\overline{\mathbb{Q}\left(f_{H, q}(p)\right)}=\overline{\mathbb{Q}\left(p^{\prime}, q\right)}$.

Proof. This follows from Lemma 9.2 by choosing a minimally rigid spanning subgraph $H$ of $G$ and then putting $f=f_{H, q}$ and $\mathbb{K}=\mathbb{Q}(q)$.

Given a linearly constrained framework $(G, p, q)$ in $\mathbb{R}^{2}$, we say that two vertices $u, v$ of $G$ are globally linked in $(G, p, q)$ if $\|p(u)-p(v)\|=\left\|p^{\prime}(u)-p^{\prime}(v)\right\|$ whenever $\left(G, p^{\prime}, q\right)$ is equivalent to $(G, p, q)$.

Lemma 9.4. Let $(G, p, q)$ be a generic linearly constrained framework in $\mathbb{R}^{2}$ and $v$ be a node of $G$ such that $N_{G}(v)=\{u, w, x\}$ and $G-v$ is rigid. Then $u, w$ are globally linked in $(G, p, q)$. 
The proof of Lemma 9.4 is the same as that of [15, Lemma 4.1]. The only difference being that we use Lemma 9.3 instead of [15, Lemmas 3.3, 3.4].

Lemma 9.5. Suppose $G$ is a rigid, $\mathcal{M}_{l c}$-connected looped simple graph and $\{u, v\}$ is an unbalanced 2-separation in $G$. Then $u, v$ are globally linked in every generic realisation of $G$ as a linearly constrained framework in $\mathbb{R}^{2}$.

Proof. We use induction on $|E(G)|$. Choose an unbalanced 2-separation $\left(H_{1}, H_{2}\right)$ of $G$ such that $H_{2}$ is simple, $u, v \in V\left(H_{1}\right)$ and $\left|V\left(H_{2}\right)\right|$ is as small as possible. Let $V\left(H_{1}\right) \cap V\left(H_{2}\right)=$ $\left\{u^{\prime}, v^{\prime}\right\}$. Then $H_{2}+u^{\prime} v^{\prime}$ is 3-connected. In addition $H_{1}+u^{\prime} v^{\prime}$ is rigid, and $H_{1}+u^{\prime} v^{\prime}$ and

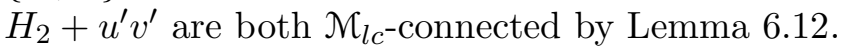

Suppose $H_{2}+u^{\prime} v^{\prime} \neq K_{4}$. By Theorem 6.11, $H_{2}+u^{\prime} v^{\prime}$ has an admissible edge $e$ distinct from $u^{\prime} v^{\prime}$ or an admissible node $w$ distinct from both $u^{\prime}$ and $v^{\prime}$. Let $H_{2}^{\prime}=H_{2}-e$ in the former case and otherwise let $H_{2}^{\prime}=H_{2}-w+x y$ be be obtained by performing an admissible

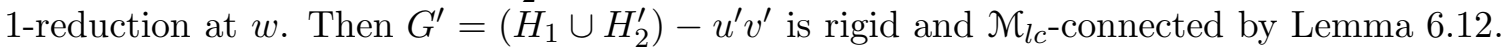
By induction, $u, v$ are globally linked in every generic realisation of $G^{\prime}$. This immediately implies that $u, v$ are globally linked in every generic realisation of $G$ if $G=G^{\prime}+f$. Hence we may suppose that $G=G^{\prime}-w+x y$. Since $G$ is redundantly rigid and $w$ is a node of $G, G-w$ is rigid. We can now use Lemma 9.4 to deduce that $x, y$ are globally linked in $G$. The fact that $u, v$ are globally linked in every generic realisation of $G^{\prime}$ now implies that $u, v$ are globally linked in every generic realisation of $G$.

It remains to consider the case when $H_{2}+u^{\prime} v^{\prime}=K_{4}$. Choose $w \in V\left(H_{2}\right) \backslash\left\{u^{\prime}, v^{\prime}\right\}$. Since $G$ is redundantly rigid and $w$ is a node of $G, G-w$ is rigid. Lemma 9.4 now implies that $u^{\prime}, v^{\prime}$ are globally linked in every generic realisation of $G$. If $\{u, v\}=\left\{u^{\prime}, v^{\prime}\right\}$ then we are done so we may assume this is not the case. Then $\{u, v\}$ is an unbalanced 2-seperation of $H_{1}+u^{\prime} v^{\prime}$. Since $H_{1}+u^{\prime} v^{\prime}$ is rigid and $\mathcal{M}_{l c}$-connected by Lemma 6.12, we may use induction to deduce that $u, v$ are globally linked in every generic realisation of $H_{1}+u^{\prime} v^{\prime}$. The fact that $u^{\prime}, v^{\prime}$ are globally linked in every generic realisation of $G$, now implies that $u, v$ are globally linked in every generic realisation of $G$.

Proof of Theorem 9.1. We use induction on $b(G)$. If $b(G)=0$ then the result follows from Lemma 8.1 and Theorem 8.3 . Hence we may suppose that $b(G) \geq 1$. Let $\left(H_{1}, H_{2}\right)$ be an unbalanced 2-separation in $G$ where $H_{2}$ is loopless and $\left|V\left(H_{2}\right)\right|$ is as small as possible. Let $V\left(H_{1}\right) \cap V\left(H_{2}\right)=\{u, v\}$. Then $H_{2}+u v$ is 3-connected and we have $b(G)=b\left(H_{1}+\right.$ $u v)+1$ by Lemma 7.2. In addition, $H_{1}+u v$ is rigid, and $H_{1}+u^{\prime} v^{\prime}$ and $H_{2}+u^{\prime} v^{\prime}$ are both $\mathcal{M}_{l c}$-connected by Lemma 6.12. Since $\left(H_{2}+u v,\left.p\right|_{H_{2}}\right)$ is globally rigid as a bar-joint framework by Theorem 1.1 and $u, v$ are globally linked in $(G, p, q)$ by Lemma 9.5, the number of linearly constrained frameworks which are equivalent to $(G, p, q)$ is exactly twice the number of linearly constrained frameworks which are equivalent to $\left(H_{1}+u v,\left.p\right|_{H_{1}},\left.q\right|_{H_{1}}\right)$ (each equivalent framework to $\left(H_{1}+u v,\left.p\right|_{H_{1}},\left.q\right|_{H_{1}}\right)$ gives rise to two equivalent frameworks to $(G, p, q)$ which are related by reflecting $H_{2}$ in the line through $\left.u, v\right)$. We can now use induction to deduce that the number of linearly constrained frameworks which are equivalent to $(G, p, q)$ is $2 \times 2^{b\left(H_{1}+u v\right)}=2^{b(G)}$.

We close by noting that the problem of counting the number of non-congruent frameworks which are equivalent to a given generic bar-joint framework in $\mathbb{R}^{2}$ can be converted to that of counting the number of distinct frameworks which are equivalent to a related linearly constrained framework in $\mathbb{R}^{2}$. Given a simple graph $G$ we construct a looped simple graph $G^{*}$ by choosing an edge $u v$ of $G$ and adding two loops at both $u$ and $v$. It is not difficult to see that the number of distinct linearly constrained frameworks which are equivalent to a generic rigid $\left(G^{*}, p, q\right)$ is exactly twice the number of non-congruent bar-joint framework 
frameworks which are equivalent to $(G, p)$. In particular, we can use this construction to deduce Theorem 1.1 from Theorem 9.1 .

Acknowledgements. The second and third authors would like to thank the London Mathematical Society for providing partial financial support for this research through a scheme 4 grant.

\section{REFERENCES}

[1] A. Berg And T. Jordan, A Proof of Connelly's Conjecture on 3-connected Circuits of the Rigidity Matroid, Journal of Combinatorial Theory, Series B 88, (2003), 77-97.

[2] A. R. Berg ANd T. Jordán, Algorithms for graph rigidity and scene analysis, in Algorithms - ESA 2003, volume 2832 of Lecture Notes in Comput. Sci., pages 7889. Springer, Berlin, 2003.

[3] K. Clinch, Global rigidity of 2-dimensional direction-length frameworks with connected rigidity matroids, arxiv preprint 1608.08559 .

[4] R. Connelly, On generic global rigidity, Applied geometry and discrete mathematics, 147-155, DIMACS Ser. Discrete Math. Theoret. Comput. Sci., 4, Amer. Math. Soc., Providence, RI, 1991.

[5] R. Connelly, Generic global rigidity, Discrete and Computational Geometry 33 (2005), 549-563.

[6] R. Connelly and W. Whiteley, Global rigidity: the effect of coning, Discrete and Computational Geometry 434 (2010) 717-735

[7] C.R. Coullard and L. Hellerstein, Independence and port oracles for matroids, with an application to computational learning theory, Combinatorica 16 (1996), no. 2, 189-208.

[8] J. Cruickshank, H. Guler, B. Jackson and A. Nixon, Rigidity of linearly constrained frameworks, International Mathematics Research Notices, to appear 2018.

[9] Y. Eftekhari, B. Jackson, A. Nixon, B. Schulze, S. Tanigawa and W. Whiteley, Pointhyperplane frameworks, slider joints, and rigidity preserving transformations, Journal of Combinatorial Theory: Series B, to appear 2019.

[10] B. Hendrickson, Conditions for unique graph realizations SIAM Journal on Computing 21 (1992), no. $1,65-84$

[11] B. Jackson and T. Jordán, Connected Rigidity Matroids and Unique Realisations of Graphs, Journal of Combinatorial Theory: Series B 94 (2005) 1-29.

[12] B. Jackson And T. Jordán, The d-Dimensional Rigidity Matroid of Sparse Graphs, Journal of Combinatorial Theory: Series B 95, (2005) 118-133.

[13] B. JaCKSON AND T. Jordán, Graph theoretical techniques in the analysis of uniquely localizable sensor networks, in Localization Algorithms and Strategies for Wireless Sensor Networks, Guoqiang Mao and Baris Fidan eds., IGI Global, 2009, 146-173.

[14] B. JACKSON AND T. JoRDÁn, Globally rigid circuits of the direction-length rigidity matroid, Journal of Combinatorial Theory: Series B 100, (2010) 1-22.

[15] B. JaCKSON, T. Jordán, AND Z. SzabadKA, Globally linked pairs of vertices in equivalent realizations of graphs, Discrete and Computational Geometry, Vol. 35, 493-512, 2006.

[16] B. Jackson, T. McCourt And A. Nixon, Necessary conditions for the generic global rigidity of frameworks on surfaces, Discrete and Computational Geometry, 52:2 (2014) 344-360.

[17] B. JaCkson And A. Nixon, Stress matrices and global rigidity of frameworks on surfaces, Discrete and Computational Geometry 54:3 (2015), 586-609.

[18] T. Jordán, C. Kiraly and S. Tanigawa, Generic global rigidity of body-hinge frameworks, Journal of Combinatorial Theory: Series B 117 (2016) 59-76.

[19] A. Lee And I. Streinu, Pebble game algorithms and sparse graphs, Discrete Mathematics, 308 (2008), no. $8,1425-1437$.

[20] J.G. Oxley, Matroid Theory, Oxford Science Publications. The Clarendon Press, Oxford University Press, New York 1992. xii+532pp.

[21] J. Saxe, Embeddability of weighted graphs in k-space is strongly NP-hard, In Seventeenth Annual Allerton Conference on Communication, Control, and Computing, Proceedings of the Conference held in Monticello, Ill., October 10-12, 1979.

[22] I. Streinu And L. Theran, Slider-pinning rigidity: a Maxwell-Laman-type theorem, Discrete and Computational Geometry 44:4, (2010) 812-837.

[23] L. Theran, A. Nixon, E. Ross, M. Sadjadi, B. Servatius and M. Thorpe, Anchored boundary conditions for locally isostatic networks, Physical Review E 92:5 (2015) 053306. 
Department of Mathematics, Faculty of Arts \& Sciences, Kastamonu University, KastaMONU, TURKEY

E-mail address: hakanguler19@gmail.com

School of Mathematical Sciences, Queen Mary University of London, Mile End Road, LonDON E1 4NS, UK.

E-mail address: b.jackson@qmul.ac.uk

Department of Mathematics and Statistics, Lancaster University, LA1 4YF, U.K.

E-mail address: a.nixon@lancaster.ac.uk 